\title{
Maternal Body Mass Index and Breastfeeding Non-Initiation and Cessation: A Quantitative Review of the Literature
}

\author{
Kyoko Nomura ${ }^{1, *} \mathbb{D}$, Sachiko Minamizono ${ }^{1}$, Kengo Nagashima ${ }^{2} \mathbb{D}$, Mariko Ono ${ }^{3}$ \\ and Naomi Kitano ${ }^{4}$ (D) \\ 1 Department of Environmental Health Science and Public Health, Akita University Graduate School of \\ Medicine, Akita 010-8543, Japan; sachikot@med.akita-u.ac.jp \\ 2 Research Center for Medical and Health Data Science, The Institute of Statistical Mathematics, \\ Tokyo 190-8562, Japan; nshi@ism.ac.jp \\ 3 Teikyo University School of Medicine, Tokyo 173-8605, Japan; malinkopf@gmail.com \\ 4 Research Center for Community Medicine and Department of Public Health, Wakayama Medical University \\ School of Medicine, Wakayama 641-8509, Japan; naomiuk@wakayama-med.ac.jp \\ * Correspondence: knomura@med.akita-u.ac.jp; Tel.: +81-018-884-6087
}

Received: 5 August 2020; Accepted: 31 August 2020; Published: 2 September 2020

\begin{abstract}
This study aims to investigate which maternal body mass index (BMI) categories are associated with the non-initiation or cessation of breastfeeding (BF) based on a quantitative review of the literature. We searched Ovid MEDLINE and EBSCO CINAHL for peer-reviewed articles published between 1946 (MEDLINE) or 1981 (CINAHL), and 2019. Selected studies were either cross-sectional or cohort studies, of healthy mothers and infants, that reported nutrition method (exclusive/full or any) and period (initiation/duration/cessation) of breastfeeding according to maternal BMI levels. Pairwise meta-analyses of 57 studies demonstrated that the pooled odds risks (OR) of not initiating BF among overweight and obese mothers compared to normal weight mothers were significant across 29 (OR 1.33, 95\% confidence interval (CI), 1.15-1.54, $\mathrm{I}^{2}=98 \%$ ) and 26 studies (OR 1.61, 95\% CI, 1.33-1.95, $\mathrm{I}^{2}=99 \%$ ), respectively; the pooled risks for BF cessation were inconsistent in overweight and obese mothers with substantial heterogeneity. However, we found that overweight mothers $(n=10$, hazard ratio (HR) $1.16,95 \% \mathrm{CI}, 1.07-1.25 ; \mathrm{I}^{2}=23 \%$ ) and obese mothers ( $n=7$, HR 1.45, 95\% CI: $1.27-1.65$; $\mathrm{I}^{2}=44 \%$ ) were both associated with an increased risk of not continuing any BF and exclusive BF, respectively. Overweight and obese mothers may be at increased risk of not initiating or the cessation of breastfeeding.
\end{abstract}

Keywords: breastfeeding non-initiation/cessation; exclusive/any breastfeeding; maternal BMI; meta-analysis

\section{Introduction}

Breastfeeding has overwhelming positive evidence for both infants and mothers. Breast milk consists of bioactive factors that play a role in immunological strengthening [1], carcinogenic development [2], neural and psychological benefits [3], and a possible protective factor for obesity in childhood [4]. In addition, recent literature demonstrates that children who are exclusively breastfed have lower infectious morbidity and mortality than those who are not breastfed, or partially breastfed [5]. Breastfeeding has a positive effect on women's health in that it can prevent breast cancer [6], diabetes [7], and ovarian cancer [8]. According to a particular article published in the Lancet in 2016, the scaling up of breastfeeding can prevent an estimated 823,000 child deaths and 20,000 breast cancer deaths every year [9]. 
Despite such important features of breastfeeding practice, the global prevalence of breastfeeding at 12 months is lower than $20 \%$ in most high-income countries, such as the UK $(<1 \%)$ [10], the USA (27\%) [11], Norway (35\%) [12], and Sweden (16\%) [13]. A variety of factors determine breastfeeding non-initiation and cessation. These include biological factors such as maternal characteristics or delivery outcome, social and environmental factors, and even the perception of infant feeding preference of the parents and family [9]. Among these, there is also a growing set of studies from Western countries showing that maternal obesity might be associated with breastfeeding failure [14].

Nevertheless, these studies are still inconsistent due to the differences in ethnicity, population, body size classification, study design, sample size, risk measurement used, and confounder adjustment. Moreover, these studies hardly investigated the effect of being underweight on breastfeeding outcomes. Unlike Western countries where obesity is prevalent, underweight women of reproductive age in Asian countries [15] are seriously concerned about the health consequence of their infants (i.e., low weight infants) and the adverse effects on breastfeeding [16].

Hence, the purpose of this study was to investigate which maternal body mass index (BMI) categories (i.e., underweight or obesity) are associated with non-initiation or cessation of breastfeeding based on quantitative reviews.

\section{Methods}

The review followed the Preferred Reporting Items for Systematic Reviews and Meta-Analyses (PRISMA) [17].

\subsection{Literature Search}

A literature search was performed of the Ovid MEDLINE and EBSCO CINAHL databases from January 1946 and January 1981, respectively, through 8 June 2019 by using key terms (All Fields) of breastfeeding ("breastfeeding" or "breast feeding" or "breastfed" or "breastfeed" or "breast milk" or "human milk" or "lactation") combined with type of breastfeeding ("exclusive" or "full" or "any") and initiation or duration of breastfeeding ("initiation" or "duration" or "cessation") and ("body mass index" or "BMI"). The search strategy is presented in Table S1 with the number of each search result according to the search term. The search process was initially confirmed by Osaka University Life Science Library and repeatedly updated by 3 authors (KN, NK, and SM) to ensure a reliable reference collection.

\subsection{Selection Criteria}

Studies meeting the following criteria qualified for inclusion in our meta-analysis: (i) the study was published as original research in any language; (ii) the study did not merely report proportions or differences, but a risk (i.e., odds/risk/hazard ratio; OR, RR, HR, respectively) of breastfeeding initiation/duration/cessation among overweight/obese and/or underweight women compared to normal weight women; (iii) breastfeeding was inclusive of any breastfeeding or/and exclusive breastfeeding (i.e., if infants were given no solid food or juice/water) that included full breastfeeding; (iv) breastfeeding cessation included disrupted lactation, undesired weaning, or delayed onset of lactogenesis; and (v) the study investigated maternal BMI in which the categories were clearly presented with a value range, or defined according to guidelines such as the World Health Organization (WHO) classification [18], the WHO Asian-specific pre-pregnancy body mass index [19], and the Institute of Medicine [20]. The WHO [18] classifies BMI into the following categories: underweight $\left(<18.5 \mathrm{~kg} / \mathrm{m}^{2}\right)$, normal weight (18.5-24.9 kg/m²), overweight $\left(25.0-29.9 \mathrm{~kg} / \mathrm{m}^{2}\right)$, and obese $\left(\geq 30.0 \mathrm{~kg} / \mathrm{m}^{2}\right)$. The Asian-specific classification differs slightly from the original and defines overweight $\left(23.0-24.9 \mathrm{~kg} / \mathrm{m}^{2}\right)$, obese I $\left(25.0-29.9 \mathrm{~kg} / \mathrm{m}^{2}\right)$, and obese II $\left(\geq 30 \mathrm{~kg} / \mathrm{m}^{2}\right)$. The Institute of Medicine [20] classifies BMI into underweight $\left(<19.1 \mathrm{~kg} / \mathrm{m}^{2}\right)$, normal weight $\left(19.1-26.0 \mathrm{~kg} / \mathrm{m}^{2}\right)$, overweight $\left(26.1-29.0 \mathrm{~kg} / \mathrm{m}^{2}\right)$, and obese $\left(\geq 29.0 \mathrm{~kg} / \mathrm{m}^{2}\right)$. 
We excluded studies if: (i) the sample size of BMI categories was not provided; (ii) they investigated BMI but did not report BMI values and ranges; (iii) they investigated "intention" of breastfeeding practice only or did not investigate the actual status of breastfeeding; (iv) they investigated the relationship of diabetic mothers, because these mothers are more likely to modify their BMI by treatment or genetic reasons; ( $v$ ) they investigated the risk of maternal BMI as a continuous variable (i.e., risk per one unit increase of pre-pregnancy BMI); (vi) the study had the same sample population of a study that had already been included in our meta-analyses; and (vii) the study had particularly focused on the very obese (i.e., obese class II, III) because such studies were too few to be included in the meta-analyses. Among these, there were 10 studies [21-30] which used continuous BMI, as provided in the Table S2.

\subsection{Data Extraction}

After screening the titles and abstracts of the retrieved papers, two investigators (KN and NK) initially and independently read the relevant papers that passed the first stage of selection. Then, SM further reviewed all relevant papers, assessed eligibility, and resolved any disagreements if necessary. The extracted data included author (year), participants, country, ethnicity, BMI classification, the timing of BMI measurement, breastfeeding (BF) initiation/cessation, timing of risk assessment used for meta-analyses, BF prevalence, measure of association, and adjusted variables to estimate. Then, one investigator $(\mathrm{KN})$ created a datasheet for analyses and computerized the relevant information, which was double checked by the statistician (Kengo Nagashima).

\subsection{Outcome of Breastfeeding}

The outcome of interest in our meta-analyses was any or exclusive breastfeeding (ABF and EBF, respectively) in relation to non-initiation or cessation. According to the definition proposed by the WHO [31], children were considered to be exclusively breastfed if they were given no other food, drink, added nonhuman milk or infant formula, except breast milk. ABF was considered if the child received liquids like water-based drinks, fruit juice, and ritual fluids, other than breast milk [31], or if the study did not clearly describe exclusive or any. We collected either ORs or HRs of BF non-initiation or cessation and, if the study provided risks of BF initiation or continuation, we recalculated the risk of $\mathrm{BF}$ non-initiation or cessation by taking the inverse of the risk of the BF status to align the direction of the risk. To estimate the pooled effect size of the risk measures, we used risk ratios in multivariable analyses rather than unadjusted models.

\subsection{Quality Assessment}

We rated the methodological quality of the studies according to a modified version of the criteria provided by the Newcastle-Ottawa scale for cohort studies [32] because our included studies were prospective, retrospective cohort, or cross-sectional studies. The Newcastle-Ottawa Scale uses a star system (with a maximum of 9 stars) to evaluate a study in 3 domains: selection of participants (4 items; Representativeness of the Exposed Cohort, Selection of the Non-Exposed Cohort, Ascertainment of Exposure, Pre-Pregnancy BMI Measurement), comparability of study groups ( 2 items; Control for Confounders), and the ascertainment of outcomes of interest (3 items; Assessment of Breastfeeding, Was Follow-Up Long Enough for Outcomes to Occur?, Adequacy of Follow-Up of Cohorts).

Representativeness of the Exposed Cohort earns one star if the representativeness of exposed individuals in the particular community is described or if the sample size is larger than 1000 . Selection of the Non-Exposed Cohort earns one star if only the normal weight group was defined as a reference group. Ascertainment of Exposure earns one star if maternal weight and height were measured and not self-reported to calculate maternal BMI. Pre-Pregnancy BMI Measurement earns one star if the study clearly stated a BMI measurement in the earlier timing of pregnancy. This indicates that the study is prospective and breastfeeding was not present at the start of the study. 
A maximum of 2 points can be given for comparability. If a study controls at least 2 variables, including maternal age, gestational week and delivery mode to calculate a risk of BF non-initiation or cessation, it will receive one point. If the study also controls for other confounding factors (i.e., socio-economic factors, psychological factors, and social support), it will receive another point.

The assessment of breastfeeding earns one star if initiation or continuation of the particular type of breastfeeding (i.e., exclusive, full, dominant, or any breastfeeding) is clearly defined. "Was Follow-Up Long Enough for Outcomes to Occur?" earns one star if the study calculated the risk estimates at a minimum of one month for initiation and 6 months for continuation, because the WHO recommends 6 months of breastfeeding. Adequacy of Follow-Up of Cohorts earns one star if the study was a cohort study and $70 \%$ of the initially enrolled sample was followed up, and N/A if the study was a cross-sectional study.

With a maximum of 9 stars, we judged studies that received a score of 9 stars to be at low risk of bias, studies that received 7 (for cross-sectional studies) or 8 stars (for cohort studies) to be at medium risk, and those that received 6 (for cross-sectional studies) or 7 stars (for cohort studies) (or fewer) to be at high risk of bias.

\subsection{Data Analysis}

Pairwise meta-analyses were performed to compare the estimate of BF non-initiation, and ABF or EBF cessation between each pre-pregnancy BMI category (i.e., underweight, normal weight as reference, overweight, and obesity). Pooled ORs and HRs and 95\% confidence intervals (CIs) of being underweight, overweight, or obese on an effect of (i.e., above 1) breastfeeding non-initiation/cessation and were estimated using random-effects models with the Sidik-Jonkman estimator [33]. Subgroup analyses were conducted according to $\mathrm{BF}(\mathrm{ABF})$ or $\mathrm{EBF}$, the timing of the risk assessment, ethnicity, and quality score ( $\leq 5 \mathrm{vs.} \geq 6$ ). For a study reporting only subgroup results, we first estimated the combined ORs or HRs within the study by using random-effects models, and then combined these with other findings from the selected study [34]. Meta-regression analyses were also performed to examine the impact of categorical variables on study effect size using regression-based techniques. Heterogeneity across individual studies by Cochran's $Q$ (a measure of weighted squared deviations), the $\mathrm{I}^{2}$ statistics (the ratio of true heterogeneity to total observed variation), and $\mathrm{Tau}^{2}$ (the variance of the true effect sizes) were estimated. $\mathrm{Tau}^{2}$ can be viewed as a point estimate of the among-study variance of true effects, while $\mathrm{I}^{2}$ would be a measure of inconsistency and the proportion of variability in the point estimates [35]. For Cochran's $Q$ test, the $P$ value indicating significant heterogeneity was set at less than 0.10 [36]. For $\mathrm{I}^{2}$, values less than $25 \%$, from $25 \%$ to $50 \%$, and more than $50 \%$ indicated modest, moderate and substantial heterogeneity, respectively. To assess publication bias, we constructed funnel plots and tested the asymmetry using Egger's and Begg's test. If Begg's test was significant, we further constructed a trim and fill funnel plot by imputing missing studies to adjust for publication bias.

All the analyses were conducted in the statistical software package STATA version 16. $p<0.05$ was considered statistically significant, unless otherwise specified.

\section{Results}

\subsection{Search Result and Selection of Studies}

Our literature search was performed on MEDLINE and CINAHL and 427 publications were identified. Of these, 122 were duplicated and excluded. We initially screened 305 papers by title and abstract search and excluded 230 papers. After adding 34 articles identified from manual searches and reviews of bibliographies of relevant studies, we critically reviewed 109 articles. After excluding 14 studies with different topics, we reviewed 95 articles for data extraction. We further excluded another 38 articles due to extreme obesity $(n=2)$ [37,38], pre-eclampsia $(n=1)$ [39], duplicate data sources $(n=1)$ [40], gestational diabetes mellitus $(\mathrm{GDM})(n=4)$ [41-44], inability to integrate $(n=20)$ [45-64] 
and continuous BMI $(n=10)$ [21-30], resulting in 57 articles that were included in the meta-analyses (Figure 1).

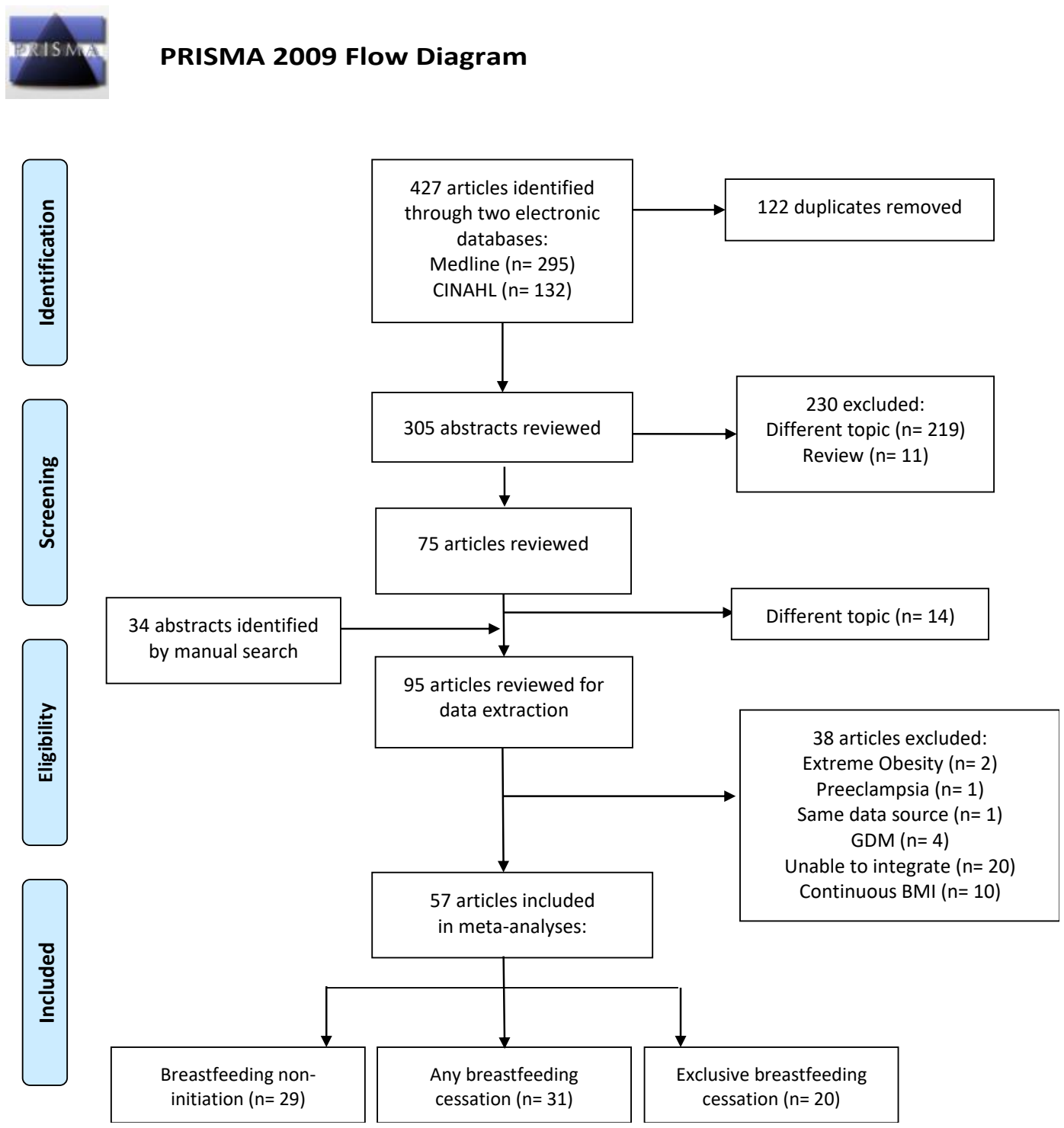

Figure 1. Selection of studies published from 1946 (MEDLINE) and 1981 (CINAHL) to 2019 and included in a meta-analysis of maternal BMI and breastfeeding practice. GDM-gestational diabetes mellitus.

\subsection{Study Characterisics}

The characteristics of the 57 included articles are presented in Table 1. Among 57 studies, 43 were published in the US and European countries [65-107], eight in Australia [60,108-114], three in China and Japan [16,115,116], two in Kuwait and Iran [117,118] and one in Brazil [119]. Except for one study [65], all were published after the year 2000, of which 15 [60,66-75,108-111] were published between 2000 and 2009, and 41 studies were published between 2010 and 2019. The majority of the included studies reported adjusted risk of BF non-initiation or cessation, except for three studies $[80,113,117]$. Twenty-nine studies $[16,60,65-67,70,73,74,76-79,83,86,88,89,96-99,104-107,110-$ $112,114,118]$ investigated BF initiation by a logistic regression model. Forty studies $[67-73,75,76,80-82$, 84,85,87,90-97,100-104,106,108-111,113-119] investigated BF cessation by either a logistic regression model or a Cox proportional hazard model or both, of which 14 studies $[67,69-71,75,81,82,87,92,94$, 109-111,117] investigated BF continuation for six months or longer. 
Table 1. The characteristics of the 57 included articles.

\begin{tabular}{|c|c|c|c|c|c|c|c|c|c|c|c|c|}
\hline \multirow{2}{*}{$\begin{array}{l}\text { Author } \\
\text { (Year) }\end{array}$} & \multirow[t]{2}{*}{ Participants } & \multirow[t]{2}{*}{ Country } & \multirow[t]{2}{*}{ Ethnicity } & \multirow[t]{2}{*}{ BMI } & \multirow[t]{2}{*}{ BF } & \multirow{2}{*}{$\begin{array}{l}\text { Initiation or } \\
\text { Cessation for } \\
\text { Meta-Analyses }\end{array}$} & \multicolumn{2}{|c|}{$\begin{array}{l}\text { Timing of Risk Ratio } \\
\text { Included in } \\
\text { Meta-Analyses }\end{array}$} & \multirow{2}{*}{$\begin{array}{l}\text { BF Proportion (if } \\
\text { Relevant Timing } \\
\text { Available) }\end{array}$} & \multirow[t]{2}{*}{$\begin{array}{l}\text { Measure of } \\
\text { Association }\end{array}$} & \multirow{2}{*}{$\begin{array}{l}\text { Adjusted Variables to Estimate } \\
\text { Risk of Breastfeeding } \\
\text { Non-Initiation or Cessation }\end{array}$} & \multirow[t]{2}{*}{ Quality } \\
\hline & & & & & & & Initiation & Cessation & & & & \\
\hline $\begin{array}{c}\text { Hilson } \\
\text { (1997) [65] }\end{array}$ & 810 & US & С $99 \%$ & $\begin{array}{c}<26.1 \text { (ref), } \\
26.1-29, \geq 29.1\end{array}$ & $\mathrm{ABF} / \mathrm{EBF}$ & Initiation & Discharge & - & $75 \%$ (at delivery) & $\mathrm{OR} / \mathrm{HR}$ & $\begin{array}{c}\text { P, GW, BW, Age, Edu, } \\
\text { Participation in WIC, PCAP, DML, } \\
\text { DM, Smk }\end{array}$ & 4 \\
\hline $\begin{array}{c}\text { Donath and } \\
\text { Amir (2000) } \\
\text { [108] }\end{array}$ & $\begin{array}{l}1991 \text { mothers } \\
\text { who had } \\
\text { children under } \\
\text { age } 4 \text { years }\end{array}$ & Australia & - & $\begin{array}{l}<25 \text { (ref), } 25-30, \\
\quad \geq 30.1\end{array}$ & $\mathrm{ABF}$ & Cessation & - & $\mathrm{N} / \mathrm{A}$ & $\begin{array}{l}87 \% \text { (ever } \\
\text { breastfed) }\end{array}$ & $\mathrm{HR}$ & Age, Edu, Smk, Mar, SC, Housing & 4 \\
\hline $\begin{array}{c}\text { Sebire } \\
(2001)[66]\end{array}$ & 287,213 & UK & W $72 \%$ & $\begin{array}{c}20-25 \text { (ref), } 25-30, \\
\geq 30.1\end{array}$ & $\mathrm{ABF}$ & Initiation & Discharge & - & - & OR & R/Eth, P, Age, HTN, DM & 3 \\
\hline $\begin{array}{l}\text { Kugyelka } \\
\text { (2004) [67] }\end{array}$ & В 263, H 235 & US & B $53 \%, \mathrm{H} 47 \%$ & $\begin{array}{l}<19.1-26.0 \text { (ref), } \\
26.1-29, \geq 29.1\end{array}$ & $\mathrm{ABF} / \mathrm{EBF}$ & Initiation/Cessation & Discharge & 6 months & $\begin{array}{c}\text { Black } 45 \%, \\
\text { Hispanic } 59 \% \\
\text { (ever breastfed in } \\
\text { hospital) }\end{array}$ & $\mathrm{OR} / \mathrm{HR}$ & Age, Edu, P, GW, BW, Smk, DLM & 6 \\
\hline $\begin{array}{l}\text { Hilson } \\
\text { (2004) [68] }\end{array}$ & 151 & US & $\mathrm{W}$ & $\begin{array}{c}<26.0 \text { (ref), } \\
26.1-29, \geq 29.1\end{array}$ & $\mathrm{ABF}$ & Cessation & - & N/A & 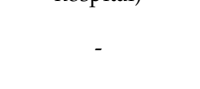 & $\mathrm{HR}$ & $\begin{array}{l}\text { PDB, RTW, Lower satisfaction of } \\
\text { appearance, Greater indifference } \\
\text { towards breastfeeding }\end{array}$ & 4 \\
\hline $\begin{array}{l}\text { Grijibovski } \\
\text { (2005) [69] }\end{array}$ & 1078 & Russia & Russian & $\begin{array}{l}\text { Underweight, } \\
\text { Normal (ref), } \\
\text { Overweight }\end{array}$ & $\mathrm{ABF}$ & Cessation & - & 12 months & $\begin{array}{c}96 \% \text { (1 month) } \\
18 \% \text { (12 months })\end{array}$ & OR & $\begin{array}{l}\text { Age, Edu, O, Mar, DLM, GW, BW, } \\
\text { InfSex, FTIPC, P }\end{array}$ & 6 \\
\hline $\begin{array}{c}\text { Forster } \\
\text { (2006) [109] }\end{array}$ & 764 & Australia & Aus $70 \%$ & $\begin{array}{l}<20,20-25 \text { (ref), } \\
26-29, \geq 29.1\end{array}$ & $\mathrm{ABF}$ & Cessation & - & 6 months & $55 \%$ (6 moths) & OR & $\begin{array}{l}\text { Age, PIFI, Desire, PH, Smk, RH, } \\
\text { ROB, PCAP, AOD, RP }\end{array}$ & 4 \\
\hline $\begin{array}{c}\text { Hilson } \\
\text { (2006) [70] }\end{array}$ & $\begin{array}{l}876 \text { who gained } \\
\text { weight within } \\
\text { the IOM } \\
\text { recommendation }\end{array}$ & US & $\mathrm{W}$ & $\begin{array}{l}<19.8,19.8-26.0 \\
(\mathrm{ref}), 26.1-29.0 \\
\quad \geq 29.1\end{array}$ & $\mathrm{ABF}$ & Initiation/Cessation & Discharge & 24 months & $91 \%$ (initiated) & $\mathrm{OR} / \mathrm{HR}$ & $\begin{array}{l}\text { Edu, Smk, Age, P, Participation in } \\
\text { WIC/PCAP, DLM }\end{array}$ & 5 \\
\hline $\begin{array}{l}\text { Oddy (2006) } \\
{[1110]}\end{array}$ & 1803 & Australia & C $86 \%$ & $\begin{array}{c}<25 \text { (ref), } 25-29.9, \\
\geq 30\end{array}$ & $\mathrm{ABF}$ & Initiation/Cessation & 1 month & $\begin{array}{l}2,4 \text {, and } 6 \\
\text { months }\end{array}$ & $\begin{array}{l}91 \%(1 \text { month }), \\
74 \% \text { ( } 2 \text { months }), \\
59 \% \text { (4 months), } \\
49 \% \text { ( } 6 \text { months })\end{array}$ & $\mathrm{OR} / \mathrm{HR}$ & $\begin{array}{l}\text { InfSex, GW, BW, Edu, Age, Eth, P, } \\
\text { Smk, DLM, PB, Age solids } \\
\text { introduced }\end{array}$ & 7 \\
\hline $\begin{array}{l}\text { Scott (2006) } \\
{[600]}\end{array}$ & 556 & Australia & $\mathrm{W}$ & $\begin{array}{c}<25 \text { (ref), } 25-29.9, \\
\geq 30\end{array}$ & $\mathrm{ABF} / \mathrm{EBF}$ & Initiation & Discharge & - & $\begin{array}{c}\text { ABF } 94 \%, \text { EBF } \\
77 \%\end{array}$ & OR & $\begin{array}{l}\text { Age, MS, O (mother, father), ROB, } \\
\text { PCAP, DLM, ADM, FP (father, } \\
\text { grandmother), PH (grandmother), } \\
\text { Timing, Smk, IFS (mother) }\end{array}$ & 4 \\
\hline $\begin{array}{l}\text { Baker (2007) } \\
\quad[71]\end{array}$ & 37,459 & Denmark & Danish & $\begin{array}{l}<18.5,18.5-24.9 \\
\text { (ref), 25.0-29.9, } \\
30.0-34.9\end{array}$ & $\mathrm{ABF} / \mathrm{EBF}$ & Cessation & - & 6 months & $65 \%$ (6 months) & IRR/OR & $\begin{array}{c}\text { Age, GWG, POC, O, P, Smk, DLM, } \\
\text { PA, InfSex }\end{array}$ & 8 \\
\hline
\end{tabular}


Table 1. Cont.

\begin{tabular}{|c|c|c|c|c|c|c|c|c|c|c|c|c|}
\hline \multirow[t]{2}{*}{$\begin{array}{l}\text { Author } \\
\text { (Year) }\end{array}$} & \multirow[t]{2}{*}{ Participants } & \multirow[t]{2}{*}{ Country } & \multirow[t]{2}{*}{ Ethnicity } & \multirow[t]{2}{*}{ BMI } & \multirow[t]{2}{*}{ BF } & \multirow{2}{*}{$\begin{array}{l}\text { Initiation or } \\
\text { Cessation for } \\
\text { Meta-Analyses }\end{array}$} & \multicolumn{2}{|c|}{$\begin{array}{l}\text { Timing of Risk Ratio } \\
\text { Included in } \\
\text { Meta-Analyses } \\
\end{array}$} & \multirow{2}{*}{$\begin{array}{l}\text { BF Proportion (if } \\
\text { Relevant Timing } \\
\text { Available) }\end{array}$} & \multirow[t]{2}{*}{$\begin{array}{l}\text { Measure of } \\
\text { Association }\end{array}$} & \multirow{2}{*}{$\begin{array}{l}\text { Adjusted Variables to Estimate } \\
\text { Risk of Breastfeeding } \\
\text { Non-Initiation or Cessation }\end{array}$} & \multirow[t]{2}{*}{ Quality } \\
\hline & & & & & & & Initiation & Cessation & & & & \\
\hline $\begin{array}{l}\text { Jain (2007) } \\
{[72]}\end{array}$ & 7661 & US & Multiethnicities & $\begin{array}{c}19.8-26.0(\mathrm{ref}) \\
26.1-29.0, \geq 29.1\end{array}$ & $\mathrm{ABF}$ & Cessation & - & 10 weeks & $47 \%$ (10 weeks) & OR & BMI, GWG, Eth, Age, Edu, P & 5 \\
\hline $\begin{array}{l}\text { Donath and } \\
\text { Amir (2008) } \\
\text { [111] }\end{array}$ & 3075 & Australia & - & $\begin{array}{l}20-24.9(\mathrm{ref}) \\
25-29, \geq 29.1\end{array}$ & $\mathrm{ABF}$ & Initiation/Cessation & 1 week & 6 months & $\begin{array}{l}88 \% \text { ( } 1 \text { week), } \\
57 \% \text { (6 months) }\end{array}$ & OR & Age, Edu, Smk, ADM, SED, DLM & 7 \\
\hline $\begin{array}{c}\text { Manios } \\
(2009)[73]\end{array}$ & $\begin{array}{l}1897 \text { children } \\
\text { aged } 12 \text { to } 60 \\
\text { months }\end{array}$ & Greek & Greek & $\begin{array}{c}<19.8,19.8-26.0 \\
\text { (ref), } 26.0-29.0 \\
\geq 29.1\end{array}$ & $\mathrm{ABF}$ & Initiation/Cessation & - & N/A & - & OR & BW, GW, P, Age, Edu, Smk & 6 \\
\hline $\begin{array}{c}\text { Fairlie } \\
\text { (2009) [74] }\end{array}$ & 1436 & US & W $74 \%$ & $\begin{array}{l}20-24.9 \text { (ref), } \\
25-29.9, \geq 30\end{array}$ & $\mathrm{ABF}$ & Initiation & - & - & $86 \%$ & OR & $\begin{array}{l}\text { Age, Eth, GW, DLM, Inc, Edu, } \\
\text { ROB, POC }\end{array}$ & 5 \\
\hline $\begin{array}{l}\text { Kehler } \\
\text { (2009) [75] }\end{array}$ & 780 & Canada & Can $83 \%$ & $\begin{array}{l}<18.5,18.5-24.9 \\
\text { (ref), } 25.0-29.9 \\
\geq 30\end{array}$ & $\mathrm{ABF}$ & Cessation & - & 6 months & $72 \%$ (6 months) & OR & $\begin{array}{l}\text { RTW or intending to within first } \\
\text { postpartum year, AOD }\end{array}$ & 4 \\
\hline $\begin{array}{l}\operatorname{Liu}(2010) \\
{[76]}\end{array}$ & $\begin{array}{l}3517 \text { (White) } \\
2840 \text { (Black) }\end{array}$ & US & W 55\%, B 45\% & $\begin{array}{l}<18.5,18.5-24.9 \\
(\text { ref), } 25-29.9 \\
\quad 30-34.9\end{array}$ & $\mathrm{ABF}$ & Initiation/Cessation & $\begin{array}{l}\text { After } \\
\text { delivery }\end{array}$ & 10 weeks & $\begin{array}{c}\text { W 67\%, B 41\% } \\
\text { (after delivery); } \\
\text { W 55\%, B 42\% (10 } \\
\text { weeks) }\end{array}$ & $\mathrm{OR} / \mathrm{HR}$ & $\begin{array}{l}\text { Age, Edu, Mar, PB, Smk, BW, P, } \\
\text { FTIPC }\end{array}$ & 4 \\
\hline $\begin{array}{l}\text { Kitsantas } \\
\text { (2010) [77] }\end{array}$ & 10,700 & US & W $60 \%$ & $\begin{array}{l}<18.5,18.5-24.9 \\
(\mathrm{ref}), 25-29.9 \\
30-34.9\end{array}$ & $\mathrm{ABF}$ & Initiation & - & $\mathrm{N} / \mathrm{A}$ & $69 \%$ & OR & $\begin{array}{l}\text { Age, Mar, Eth, P, Smk, Alc, DLM, } \\
\text { InfSex, GW, BW }\end{array}$ & 5 \\
\hline $\begin{array}{l}\text { Biro (2011) } \\
\quad[112]\end{array}$ & 3352 & Australia & $\begin{array}{c}98 \% \\
\text { Non-aboriginal }\end{array}$ & $\begin{array}{c}<20,20-24.9 \text { (ref) } \\
25-29.9, \geq 30\end{array}$ & $\mathrm{ABF}$ & Initiation & $\begin{array}{c}\text { After } \\
\text { delivery }\end{array}$ & - & $\begin{array}{l}82 \% \text { (after } \\
\text { delivery) }\end{array}$ & OR & $\begin{array}{l}\text { P, Age, HC, Edu, Mar, ROB, Smk, } \\
\text { BW, ADM, BFHI accreditation, } \\
\text { MOI, Analgesia, DML, BW, ADM }\end{array}$ & 4 \\
\hline $\begin{array}{c}\text { Mehta } \\
\text { (2011) [78] }\end{array}$ & 688 & US & W 77\% & $\leq 26$ (ref), $\geq 26.1$ & $\mathrm{ABF}$ & Initiation & 3 months & - & $91 \%$ & OR & R/Eth, Edu, Mar, PS & 3 \\
\hline $\begin{array}{l}\text { Tenfelde } \\
\text { (2011) [79] }\end{array}$ & 235 & US & Mexican 42\% & $\begin{array}{c}<18.5,18.5-24.9 \\
\text { (ref), } 25.0-29.9 \\
\geq 30\end{array}$ & EBF & Initiation & 1 month & - & $23 \%$ & OR & FTIPC, RTW, PIFI, & 2 \\
\hline $\begin{array}{l}\text { Leonard } \\
\text { (2011) [80] } \\
\text { Hauff and }\end{array}$ & 2288 & US & W $85 \%$ & $\begin{array}{l}<25 \text { (ref), } 25-29.9 \\
\quad \geq 30\end{array}$ & $\mathrm{ABF}$ & Cessation & - & 2 months & $86 \%$ (2 months) & OR & - & 3 \\
\hline $\begin{array}{l}\text { Demerath } \\
\text { (2012) [81] }\end{array}$ & 239 & US & C $85 \%$ & $<25$ (ref), $\geq 25.0$ & $\mathrm{ABF}$ & Cessation & - & 6 months & 76\% (6 months) & HR & DM, AvBFP, PDB & 3 \\
\hline $\begin{array}{c}\text { Bartok } \\
\text { (2012) [82] }\end{array}$ & 672 & US & W 91\% & $\begin{array}{l}18.5-24.9 \text { (ref), } \\
25-29.9, \geq 30\end{array}$ & $\mathrm{ABF}$ & Cessation & - & 6 months & $51 \%$ (6 months) & $\mathrm{HR}$ & Edu, Mar, PDB, MRBI & 5 \\
\hline $\begin{array}{l}\text { Kronborg } \\
\text { (2012) [84] }\end{array}$ & 1442 & Denmark & Danish & $\begin{array}{l}<27 \text { (ref), } 27-31.9 \\
\quad \geq 32\end{array}$ & $\mathrm{ABF}$ & Cessation & - & 17 weeks & - & HR & Age, Duration of schooling & 3 \\
\hline $\begin{array}{l}\text { Branger } \\
(2012)[85]\end{array}$ & 239 & France & C & $<30$ (ref), $\geq 30.0$ & $\mathrm{ABF}$ & Cessation & - & - & $\begin{array}{l}89 \%(1 \text { month), } \\
27 \% \text { (6 months) }\end{array}$ & OR & $\begin{array}{l}\text { Age, Decision of BF, P, O, BW, } \\
\text { Confidence, Difficulty, Situation, } \\
\text { Support of BF, DLM }\end{array}$ & 4 \\
\hline
\end{tabular}


Table 1. Cont.

\begin{tabular}{|c|c|c|c|c|c|c|c|c|c|c|c|c|}
\hline \multirow[t]{2}{*}{$\begin{array}{l}\text { Author } \\
\text { (Year) }\end{array}$} & \multirow[t]{2}{*}{ Participants } & \multirow[t]{2}{*}{ Country } & \multirow[t]{2}{*}{ Ethnicity } & \multirow[t]{2}{*}{ BMI } & \multirow[t]{2}{*}{ BF } & \multirow{2}{*}{$\begin{array}{l}\text { Initiation or } \\
\text { Cessation for } \\
\text { Meta-Analyses }\end{array}$} & \multicolumn{2}{|c|}{$\begin{array}{c}\text { Timing of Risk Ratio } \\
\text { Included in } \\
\text { Meta-Analyses }\end{array}$} & \multirow{2}{*}{$\begin{array}{l}\text { BF Proportion (if } \\
\text { Relevant Timing } \\
\text { Available) }\end{array}$} & \multirow[t]{2}{*}{$\begin{array}{l}\text { Measure of } \\
\text { Association }\end{array}$} & \multirow{2}{*}{$\begin{array}{l}\text { Adjusted Variables to Estimate } \\
\text { Risk of Breastfeeding } \\
\text { Non-Initiation or Cessation }\end{array}$} & \multirow[t]{2}{*}{ Quality } \\
\hline & & & & & & & Initiation & Cessation & & & & \\
\hline $\begin{array}{l}\text { Perrine } \\
\text { (2012) [95] }\end{array}$ & 1457 & US & W 88\% & $\begin{array}{l}<18.5,18.5-24.9 \\
\text { (ref), } 25.0-29.9 \\
\geq 30\end{array}$ & EBF & Cessation & - & - & $45 \%$ (3 months) & OR & $\begin{array}{l}\text { Age, R/Eth, PTI ratio, Edu, P, Smk, } \\
\text { NPFWIC, DLM, Mar, Intended } \\
\text { duration of EBF, BFHI practices }\end{array}$ & 6 \\
\hline $\begin{array}{l}\text { Thompson } \\
\text { (2013) [83] }\end{array}$ & $1,161,949$ & US & H $32 \%$, B $18 \%$ & $\begin{array}{l}<18.5,18.5-24.9 \\
\text { (ref), } 25-29.9, \geq 30\end{array}$ & $\mathrm{ABF}$ & Initiation & $\begin{array}{l}\text { Immediate } \\
\text { postpartum }\end{array}$ & - & $\begin{array}{l}79 \% \text { (immediate } \\
\text { postpartum) }\end{array}$ & OR & $\begin{array}{l}\text { Age, R/Eth, Edu, MH, PNC, BW, } \\
\text { InfSex, P, GW, Birth year }\end{array}$ & 5 \\
\hline $\begin{array}{l}\text { Sipsma } \\
(2013)[86]\end{array}$ & 225 & t US & $\begin{array}{l}\text { B } 40 \%, \mathrm{H} 42 \% \\
\quad \text { O } 18 \%\end{array}$ & $\begin{array}{l}<18.5,18.5-24.9 \\
\text { (ref), } 25-29.9, \geq 30\end{array}$ & $\mathrm{ABF} / \mathrm{EBF}$ & Initiation & - & - & $\begin{array}{l}71 \%(\mathrm{ABF} \\
\text { initiated), } 16 \% \\
\text { (6 months) }\end{array}$ & OR & $\begin{array}{l}\text { Age, R/Eth, Edu, Em, NPFWIC, } \\
\text { School status, FB }\end{array}$ & 3 \\
\hline $\begin{array}{l}\text { Brown } \\
\text { (2013) [87] }\end{array}$ & 4533 & Canada & Can & $\begin{array}{l}<18.5,18.5-24.9 \\
\text { (ref), } 25-29.9, \geq 30\end{array}$ & EBF & Cessation & - & 6 months & $10 \%$ (6 months) & HR & E, Inc, Mar, Smk, P, FAS, Int, EBC & 5 \\
\hline $\begin{array}{l}\text { Gubler } \\
\text { (2013) [88] }\end{array}$ & 1893 & Swiss & C $77 \%$ & $\begin{array}{l}<18.5,18.5-24.9 \\
(\text { ref), } 25-29.9, \geq 30\end{array}$ & EBF & Initiation & Discharge & - & 68\% (discharge) & OR & $\begin{array}{l}\text { P, GWG, BW, DLM, Anesthesia, } \\
\mathrm{Hb}<9.5 \mathrm{~g} / \mathrm{dL} \text {, DSH, SN, EBC, First } \\
\text { suckling and rooming-in }\end{array}$ & 3 \\
\hline $\begin{array}{l}\text { Visram } \\
\text { (2013) [89] }\end{array}$ & 22,131 & Canada & Can & $\begin{array}{l}18.5-24.9 \text { (ref), } \\
25-29.9, \geq 30\end{array}$ & EBF & Initiation & Discharge & - & 68\% (discharge) & OR & $\begin{array}{l}\text { Age, NFI, NEL, AOR, P, First } \\
\text { trimester visit, PNC, HCP, SFGA, } \\
\text { DM, DLM, NICU admission }\end{array}$ & 5 \\
\hline $\begin{array}{l}\text { Zhu (2013) } \\
\text { [116] }\end{array}$ & 1602 & China & Asian & $\begin{array}{c}<18.3,18.4-19.6 \\
\text { (ref), } 19.7-21.2 \\
\geq 21.3\end{array}$ & $\mathrm{ABF}$ & Cessation & - & 2 months & 88\% (2 months) & OR & $\begin{array}{l}\text { Age, Edu, Inc, SS, LE, GWG, GW, } \\
\text { DLM, BF on day } 1 \text {, OL }\end{array}$ & 6 \\
\hline $\begin{array}{l}\text { Hayes } \\
\text { (2014) [90] }\end{array}$ & 8508 & US & Multiethnicities & $\begin{array}{l}<18.5,18.5-24.9 \\
\text { (ref), } 25-29.9, \geq 30\end{array}$ & EBF & Cessation & - & 8 weeks & $37 \%$ (8 weeks) & $\mathrm{RR}$ & R/Eth, Age, DLM, RTW, Dep & 6 \\
\hline $\begin{array}{c}\text { Vurbic } \\
(2014)[91]\end{array}$ & 300 & US & C & $<25$ (ref), $\geq 25.0$ & $\mathrm{ABF}$ & Cessation & - & 24 weeks? & $\begin{array}{l}70 \% \text { (abstainer), } \\
43 \% \text { (smoker) }\end{array}$ & OR & Edu, Mar & 3 \\
\hline $\begin{array}{l}\text { Stuebe } \\
\text { (2014) [92] }\end{array}$ & 2335 & US & W 81\% & $\begin{array}{l}<18.5,18.5-24.9 \\
\text { (ref), } 25-29.9, \geq 30\end{array}$ & $\mathrm{ABF}$ & Cessation & - & 12 months & $\begin{array}{c}18.6 \% \\
\text { (12 months) }\end{array}$ & OR & NPFWIC, Mar, R/Eth, Age, P, Edu & 5 \\
\hline $\begin{array}{l}\text { Hauff (2014) } \\
\text { [93] }\end{array}$ & 2423 & US & W $85 \%$ & $\begin{array}{c}18.5-24.9 \text { (ref), } \\
25-29.9, \geq 30\end{array}$ & $\mathrm{ABF} / \mathrm{EBF}$ & Cessation & - & - & $\begin{array}{l}93 \% \text { (ever } \\
\text { breastfed) }\end{array}$ & $\mathrm{HR}$ & $\begin{array}{l}\text { R/Eth, planned RTW, PBE, PIFI, } \\
\text { Mar, Smk, GW, PDB, Edu }\end{array}$ & 3 \\
\hline $\begin{array}{l}\text { Dashti } \\
\text { (2014) [117] }\end{array}$ & 345 & Kuwait & Arabian & $\begin{array}{l}<25 \text { (ref), } 25-29.9 \\
\quad \geq 30\end{array}$ & EBF & Cessation & - & 6 months & $39 \%$ (6 months) & OR & - & 3 \\
\hline $\begin{array}{c}\operatorname{Cox}(2015) \\
{[94]}\end{array}$ & 489 & US & c & $<30$ (ref), $\geq 30$ & $\mathrm{ABF} / \mathrm{EBF}$ & Cessation & - & $\begin{array}{l}26 \text { and } 52 \\
\text { weeks }\end{array}$ & $\begin{array}{c}\text { ABF } 76 \% \\
\text { (4 months), EBF } \\
5.7 \% \text { (6 months) }\end{array}$ & $\mathrm{HR}$ & $\begin{array}{l}\text { Age, MPFFP, InfSex, BW, P, ADM, } \\
\text { CA, PCAP, Edu, DLM, Inc, MS, O } \\
\text { (father and mother), BC, Room, } \\
\text { PBE, TTRC, Smk }\end{array}$ & 5 \\
\hline $\begin{array}{l}\text { Smith (2015) } \\
\quad[96]\end{array}$ & 874 & Ireland & $\begin{array}{l}74 \% \text { born in } \\
\text { Ireland }\end{array}$ & $\begin{array}{l}<18.5,18.5-24.9 \\
(\text { ref), } 25-29.9 \\
30.0-40.5\end{array}$ & $\mathrm{ABF} / \mathrm{EBF}$ & Initiation/Cessation & Discharge & $\begin{array}{l}2 \text { and } 6 \\
\text { months }\end{array}$ & $\begin{array}{c}\text { EBF } 43 \% \\
\text { (discharge), } 0.7 \% \\
\text { (6 months) }\end{array}$ & OR & $\begin{array}{c}\text { GW, DLM, BW, ADM, Duration of } \\
\text { hospital stay, Paternal BMI, Smk, } \\
\text { Edu, O, Maternal and paternal } \\
\text { R/Eth, Mar, Age }\end{array}$ & 8 \\
\hline
\end{tabular}


Table 1. Cont

\begin{tabular}{|c|c|c|c|c|c|c|c|c|c|c|c|c|}
\hline \multirow{2}{*}{$\begin{array}{l}\text { Author } \\
\text { (Year) }\end{array}$} & \multirow[t]{2}{*}{ Participants } & \multirow[t]{2}{*}{ Country } & \multirow[t]{2}{*}{ Ethnicity } & \multirow[t]{2}{*}{ BMI } & \multirow[t]{2}{*}{ BF } & \multirow{2}{*}{$\begin{array}{l}\text { Initiation or } \\
\text { Cessation for } \\
\text { Meta-Analyses }\end{array}$} & \multicolumn{2}{|c|}{$\begin{array}{l}\text { Timing of Risk Ratio } \\
\text { Included in } \\
\text { Meta-Analyses }\end{array}$} & \multirow{2}{*}{$\begin{array}{l}\text { BF Proportion (if } \\
\text { Relevant Timing } \\
\text { Available) }\end{array}$} & \multirow[t]{2}{*}{$\begin{array}{l}\text { Measure of } \\
\text { Association }\end{array}$} & \multirow{2}{*}{$\begin{array}{l}\text { Adjusted Variables to Estimate } \\
\text { Risk of Breastfeeding } \\
\text { Non-Initiation or Cessation }\end{array}$} & \multirow[t]{2}{*}{ Quality } \\
\hline & & & & & & & Initiation & Cessation & & & & \\
\hline $\begin{array}{l}\text { Winkvist } \\
\text { (2015) [97] }\end{array}$ & $\begin{array}{l}16,922 \text { who } \\
\text { gained weight } \\
\text { within the IOM } \\
\text { recommendation }\end{array}$ & Norway & $\begin{array}{l}\text { Residents in } \\
\text { Norway }\end{array}$ & $\begin{array}{l}<18.5,18.5-24.9 \\
\text { (ref), } 25-29.9, \geq 30\end{array}$ & $\mathrm{ABF} / \mathrm{EBF}$ & Initiation/Cessation & 1 week & $\begin{array}{l}4 \text { and } 6 \\
\text { months }\end{array}$ & $\begin{array}{l}\text { ABF } 81 \%(6 \\
\text { months), EBF } 14 \% \\
(6 \text { months })\end{array}$ & OR & Inc, Edu, Age, Smk, P, DM, DLM & 8 \\
\hline $\begin{array}{c}\text { Forster } \\
\text { (2015) [113] }\end{array}$ & 924 & Australia & Aus born $68 \%$ & $<30$ (ref), $\geq 30$ & $\mathrm{ABF}$ & Cessation & - & 6 months & $68 \%$ (6 months) & OR & - & 3 \\
\hline $\begin{array}{l}\text { Verret-Chalifour } \\
\text { (2015) [98] }\end{array}$ & 6592 & Canada & C $97 \%$ & $\begin{array}{c}<18.5,18.5-24.9 \\
\text { (ref), 25-29.9, } \geq 30\end{array}$ & $\mathrm{ABF}$ & Initiation & $\begin{array}{c}\text { After } \\
\text { delivery }\end{array}$ & - & $87 \%$ (in hospital) & $\mathrm{RR}$ & $\begin{array}{c}\text { Place, Year of delivery, Age, Edu, } \\
\text { Mar, P, Eth, Inc, Smk, Alc, History } \\
\text { of drug use, BH, PBE, GW, HTN, } \\
\text { InfSex, BW, APG }\end{array}$ & 6 \\
\hline $\begin{array}{l}\text { Masho } \\
\text { (2015) [99] }\end{array}$ & 95,141 & US & W $60 \%$ & $\begin{array}{c}<18.5,18.5-24.9 \\
\text { (ref), } 25-29.9, \geq 30\end{array}$ & $\mathrm{BF}$ & Initiation & $\begin{array}{c}\text { After } \\
\text { delivery }\end{array}$ & - & $83 \%$ & OR & $\begin{array}{l}\text { Edu, NPFWIC, DOU, Age, PNC, } \\
\text { Dep, Inc, Smk, PV }\end{array}$ & 4 \\
\hline $\begin{array}{l}\text { Lindau } \\
\text { (2015) [100] }\end{array}$ & 605 & Italy & C & $\begin{array}{l}<18.5,18.5-24.9 \\
\text { (ref), } 25-29.9, \geq 30\end{array}$ & EBF & Cessation & - & 6 months & $16 \%$ (4 months) & OR & - & 3 \\
\hline $\begin{array}{l}\text { Castillo } \\
\text { (2016) [119] }\end{array}$ & 4011 & Brazil & W $62 \%$ & $\begin{array}{l}<18.5,18.5-24.9 \\
(\mathrm{ref}), 25-29.9, \geq 30\end{array}$ & EBF & Cessation & - & 3 months & $27 \%$ (3 months) & $\mathrm{HR}$ & $\begin{array}{l}\text { Age, Edu, P, DM, HTN, Alc, } \\
\text { InfSex, GW, BW, DLM }\end{array}$ & 7 \\
\hline $\begin{array}{l}\text { Kair (2016) } \\
{[\text { [101] }}\end{array}$ & $\begin{array}{l}2530 \text { who had } \\
\text { late preterm } \\
\text { infants }\end{array}$ & US & W 95\% & $\begin{array}{l}\text { Underweight, } \\
\text { normal, } \\
\text { overweight, } \\
\text { obese }\end{array}$ & $\mathrm{ABF}$ & Cessation & - & 6 months & - & OR & $\begin{array}{l}\text { Age, Edu, Mar, Insurance, P, DLM, } \\
\text { NICU, BW, AOR }\end{array}$ & 7 \\
\hline $\begin{array}{l}\text { Logan } \\
\text { (2016) [102] }\end{array}$ & 856 & Germany & $\begin{array}{l}\text { German } \\
\text { nationality } \\
\quad 85 \%\end{array}$ & $\begin{array}{l}<18.5,18.5-24.9 \\
\text { (ref), } 25-29.9, \geq 30\end{array}$ & $\mathrm{ABF}$ & Cessation & - & $\begin{array}{l}4 \text { and } 6 \\
\text { weeks; } 6 \\
\text { months }\end{array}$ & $\begin{array}{l}\mathrm{ABF} 67 \% \\
\text { (6 months) }\end{array}$ & $\mathrm{HR}$ & Edu, Alc, Smk, DLM, RTW & 4 \\
\hline $\begin{array}{c}\text { Tao (2017) } \\
\text { [115] }\end{array}$ & 3196 & China & Asian & $\begin{array}{c}<18.5,18.5-23.9 \\
(\mathrm{ref}), 24-27.9, \geq 28\end{array}$ & $\mathrm{ABF} / \mathrm{EBF}$ & Cessation & - & $\begin{array}{l}1,3 \text { and } 6 \\
\text { months }\end{array}$ & $\begin{array}{c}\text { EBF } 44 \% \\
\text { (1 month), } 51.6 \% \\
\text { (3 months), } 11 \% \\
\text { (6 months) }\end{array}$ & $\mathrm{HR} / \mathrm{RR}$ & $\begin{array}{c}\text { Age, GW, BW, DLM, InfSex, GWG, } \\
\text { Eth, Edu, Inc, P, Smk, Alc, HTN, } \\
\text { DM }\end{array}$ & 8 \\
\hline $\begin{array}{l}\text { de Jersey } \\
\text { (2017) [114] }\end{array}$ & 329 & Australia & Aus born $72 \%$ & $<24.9(\mathrm{ref}), \geq 25$ & EBF & Initiation/Cessation & - & 4 months & $\begin{array}{l}82 \% \text { (discharge), } \\
40 \% \text { (4 months) }\end{array}$ & OR & Edu, ROC, DLM, & 3 \\
\hline $\begin{array}{l}\text { Tehranian } \\
\text { (2017) [118] }\end{array}$ & 593 & Iran & Arabian & $<24.9$ (ref), $\geq 25$ & $\mathrm{ABF} / \mathrm{EBF}$ & Initiation/Cessation & - & 6 months & $\begin{array}{c}\text { EBF } 93 \% \\
\text { (1 month) }\end{array}$ & OR & Age, Edu, DLM, M's opinion, O & 5 \\
\hline $\begin{array}{l}\text { Haile (2017) } \\
\quad[104]\end{array}$ & 2026 & US & W $85 \%$ & $\begin{array}{c}<18.5,18.5-24.9 \\
\text { (ref), } 25-29.9, \geq 30\end{array}$ & EBF & Initiation/Cessation & - & 3 months & $\begin{array}{l}75 \% \text { (discharge), } \\
28 \% \text { (3 months) }\end{array}$ & OR & $\begin{array}{l}\text { Age, Eth, Edu, MS, PTI ratio, DM, } \\
\text { GWG, PIFI, DLM, Smk, GW, } \\
\text { ADM, BFHI }\end{array}$ & 6 \\
\hline $\begin{array}{l}\text { Wallenborn } \\
\text { (2017) [105] }\end{array}$ & 34,854 & US & W $60 \%$ & $\begin{array}{l}<18.5,18.5-24.9 \\
\text { (ref), } 25-29.9, \geq 30\end{array}$ & $\mathrm{BF}$ & Initiation & - & - & $75 \%$ & OR & $\begin{array}{l}\text { Age, Edu, R/Eth, Inc, Insurance, } \\
\text { PNC, MedS, WIC, Stressors }\end{array}$ & 4 \\
\hline
\end{tabular}


Table 1. Cont.

\begin{tabular}{|c|c|c|c|c|c|c|c|c|c|c|c|c|}
\hline \multirow[t]{2}{*}{$\begin{array}{l}\text { Author } \\
\text { (Year) }\end{array}$} & \multirow[t]{2}{*}{ Participants } & \multirow[t]{2}{*}{ Country } & \multirow[t]{2}{*}{ Ethnicity } & \multirow[t]{2}{*}{ BMI } & \multirow[t]{2}{*}{ BF } & \multirow{2}{*}{$\begin{array}{l}\text { Initiation or } \\
\text { Cessation for } \\
\text { Meta-Analyses }\end{array}$} & \multicolumn{2}{|c|}{$\begin{array}{c}\text { Timing of Risk Ratio } \\
\text { Included in } \\
\text { Meta-Analyses }\end{array}$} & \multirow{2}{*}{$\begin{array}{l}\text { BF Proportion (if } \\
\text { Relevant Timing } \\
\text { Available) }\end{array}$} & \multirow[t]{2}{*}{$\begin{array}{l}\text { Measure of } \\
\text { Association }\end{array}$} & \multirow{2}{*}{$\begin{array}{l}\text { Adjusted Variables to Estimate } \\
\text { Risk of Breastfeeding } \\
\text { Non-Initiation or Cessation }\end{array}$} & \multirow[t]{2}{*}{ Quality } \\
\hline & & & & & & & Initiation & Cessation & & & & \\
\hline $\begin{array}{c}\text { Bjørset } \\
\text { (2018) [103] }\end{array}$ & 700 & Norway & Nor $87 \%$ & $\begin{array}{c}<18.5 \\
18.5-24.9 \text { (ref), } \\
25-29.9, \geq 30\end{array}$ & $\mathrm{ABF} / \mathrm{EBF}$ & Cessation & & $\begin{array}{l}4 \text { and } 5 \\
\text { months }\end{array}$ & $\begin{array}{c}\text { ABF } 81 \% \\
\text { (5 months), EBF } \\
52.7 \% \text { (4 months) }\end{array}$ & OR & $\begin{array}{c}\text { Age, Edu, Mar, P, Smk, APUE, } \\
\text { Degree of urbanization, APUE, } \\
\text { DOU }\end{array}$ & 4 \\
\hline $\begin{array}{l}\text { Ramji (2018) } \\
\text { [107] }\end{array}$ & 12,422 & Canada & Can & $\begin{array}{l}18.5-24.9 \text { (ref), } \\
25-29.9,30-39.9 \\
40-49.9\end{array}$ & $\mathrm{ABF}$ & Initiation & Discharge & - & 68\% (discharge) & OR & $\begin{array}{l}\text { Age, P, POC, Em, Edu, Smk, DM, } \\
\text { HTN, Anesthesia }\end{array}$ & 4 \\
\hline $\begin{array}{l}\text { Marshall } \\
\text { (2019) [106] }\end{array}$ & 140 & US & W $84 \%$ & $<24.9$ (ref), $\geq 25$ & EBF & Initiation/Cessation & 6 weeks & 6 months & $\begin{array}{l}80 \% \text { (6 weeks), } \\
66 \% \text { (6 months) }\end{array}$ & OR & Age, GWG, Smk, PDB & 5 \\
\hline $\begin{array}{l}\text { Nomura } \\
(2019)[16]\end{array}$ & 6125 & Japan & Asian & $\begin{array}{c}<18.5, \\
18.5-24.9 \text { (ref), } \\
25-29.9, \geq 30\end{array}$ & EBF & Initiation & 1 month & - & 83\% (discharge) & OR & $\begin{array}{l}\text { Age, P, DLM, Alc, smk, GW, a } \\
\text { light for date infant, } \\
\text { maternal-child separation }\end{array}$ & 6 \\
\hline
\end{tabular}

Abbreviations: ABF, Any breastfeeding; ADM, admission to special care nursery; Alc, Alcohol; AOD, Anxiety or depression problem; AOR, Area of Residence; APG, Apgar score; APUE, ability to pay unforeseen expenses of 3000 NOK; Aus, Australian; AvBFP, avoidance breastfeeding in public; B, Black; BF in Day1, Breastfeeding frequency in Day1; BF, breastfeeding; BFHI, Baby-Friendly Hospital Initiative; BH, Breast History; BMI, Body Mass Index; BW, Birth weight; C, Caucasian; Can, Canadian; Dep, Self-reported Depressive symptoms; Desire, Desire to breastfeed; DLM, Delivery method; DM, Diabetes Mellitus including gestational diabetes; DML, Duration of maternity leave; DOU, degree of urbanization; DSH, duration of stay in hospital; Em, Employment; EBC, Early breast contact; EBF, Exclusive breastfeeding including Full breastfeeding; Edu, Education; FAS, Folic acid supplementation; FB, first baby; FP, feeding preference; FTIPC, first trimester initiation of prenatal care; GW, Gestational Week; GWG, Gestational Weight Gain; $\mathrm{H}$, Hispanic; HC, Health concession card holder; HCP, HealthCare Provider; HR, hazard ratio; HTN, Hypertension including gestational hypertension; IFS, infant feeding score; InfSex, infant Sex; Inc, income; Int, intention to BF; Jpn, Japan; LE, Life event in third trimester; M's opinion, mothers' opinion of breastfeeding; Mar, Marital Status; MedS, Medicaid status during pregnancy; MH, Maternal Health; MOI, Model of institution; mos, months; MPFFP, mother's perception of father's feeding preference; MRBI, maternal rating of breastfeeding importance; N/A, Not available; NEL, Neighborhood education level; NFI, neighborhood family income; NICU, Newborn Intensive Care Unit; Nor, Norwegian; NPFWIC, Nutrition program for Women, Infant and Child; O, Occupation; OL, Onset of lactation; OR, odds ratio; P, Parity, PA, Physical activity; PB, Pregnancy Problem; PBE, Previous Breastfeeding Experience; PCAP, Prenatal Care Assistance Program; PDB, planned duration of breastfeeding; PH, Past history of being breastfed; PIFI, Prenatal infant-feeding Intention; PNC, prenatal care; POC, Presence of company including a spouse or partner during early pregnancy; PS, Poverty Status; TI ratio, poverty-to-income ratio; PV, Partner Violence; R/Eth, Race/Ethnicity; ref, reference; RH, Received formula in hospital; ROB, Region of birth; Room, Room in hospital; RP, Relationship problems; RTW, Return to work/school; SC, Social security; SED, level of socio-economic disadvantage of the geographical location of the child's household; SFGA, small for gestational age; Smk, Smoking; SN, sore nipple; SS, Social Support; timing, timing of infant feeding decision; UK, United Kingdom; US, United States; W, White; wks, weeks;WIC, Women, Infant, and Children Food and Nutrition Service/Program3.3. Quality of 57 Selected Studies. 
The quality assessment of each study according to the Newcastle-Ottawa scale [32] is illustrated, together with the number of stars awarded, in Table S3. The overall quality score ranged from three to eight, with a mean score \pm standard deviation of $4.8 \pm 1.5$. Among the 57 studies investigated, there were no studies that received a score of nine stars to be at low risk of bias. Instead, eight studies [71,96, $97,101,110,111,115,119]$ received seven/eight stars and were considered to have a medium risk of bias, and the remaining 49 studies were awarded six or fewer stars. For maternal BMI, the majority of the studies investigated BMI before pregnancy but seven studies $[84,100,101,103,108,109,111]$ investigated postpartum BMI. In addition, most of the studies collected BMI information by self-reporting of mothers and the timing of BMI measurement was not always clearly described.

\subsection{Breastfeeding Non-Initiation}

Table 2 shows the pooled risk for breastfeeding non-initiation with $95 \% \mathrm{CI}$, heterogeneity, and publication bias and Figure 2 shows a forest plot for BF non-initiation according to BMI categories. The OR for BF non-initiation was 1.03 (95\% CI: 0.85-1.23; $\mathrm{I}^{2}=88 \%, p$ for $\mathrm{Q}<0.001$, $\mathrm{Tau}^{2}=0.08$ ) among 14 studies $[16,70,73,76,83,86,88,96-99,104,105,112]$ in underweight mothers. Although Begg's test was non-significant, indicating that publication bias is less likely, a large $\mathrm{I}^{2}$ and significance of $\mathrm{Q}$ test indicated substantial heterogeneity existed among the studies. Our subgroup analyses with high quality score, BF type (ABF or EBF), timing of measurement (one month or no report), and ethnicity all showed insignificant results of the effect of being underweight on BF non-initiation (data not shown). The OR of BF non-initiation in overweight and obese mothers was 1.33 (95\% CI: 1.15-1.54; $\mathrm{I}^{2}$ $=98 \%, p$ for $\left.\mathrm{Q}<0.001, \mathrm{Tau}^{2}=0.12\right)$ among 29 studies $[16,60,65-67,70,73,74,76-79,83,86,88,89,96-99$, 104-107,110-112,114,118] and 1.61 (95\% CI: 1.33-1.95; $\mathrm{I}^{2}=99 \%, p$ for $\mathrm{Q}<0.001$, Tau ${ }^{2}=0.20$ ) among 26 studies $[16,60,65-67,70,73,74,76,77,79,83,86,88,89,96-99,104,105,107,110-112,118]$, respectively. As the $p$ value for Begg's tests in both overweight and obese mothers was less than 0.05 , indicating publication bias, we additionally performed a trim and fill funnel plot by imputing missing studies. We then observed the significance for obese (OR 1.32, 95\% CI: 1.08-1.61) but not for overweight women (OR 1.13, 95\% CI: 0.98-1.30).

Table 2. Pooled risk of breastfeeding non-initiation with 95\% confidence interval (CI), heterogeneity and publication bias.

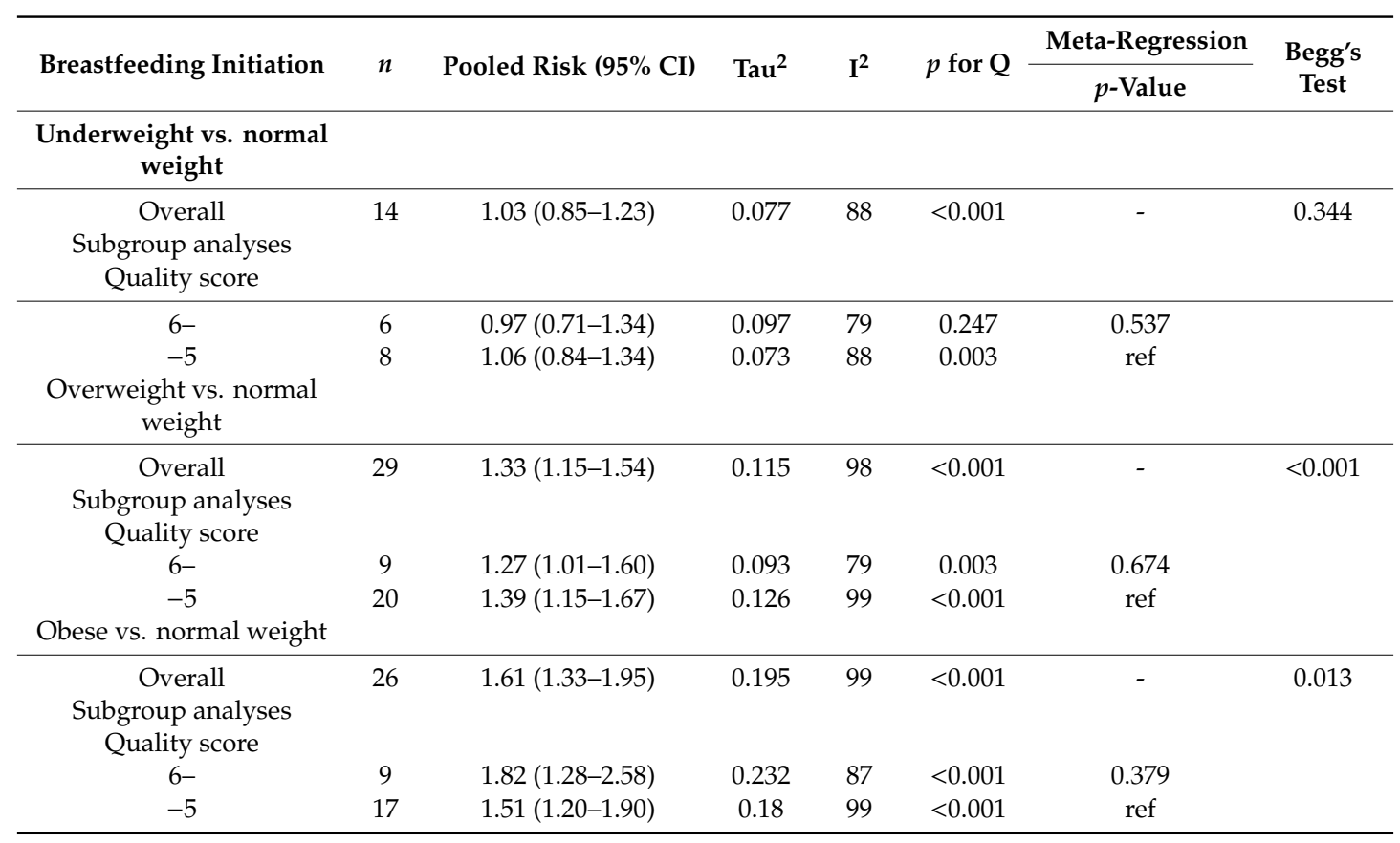




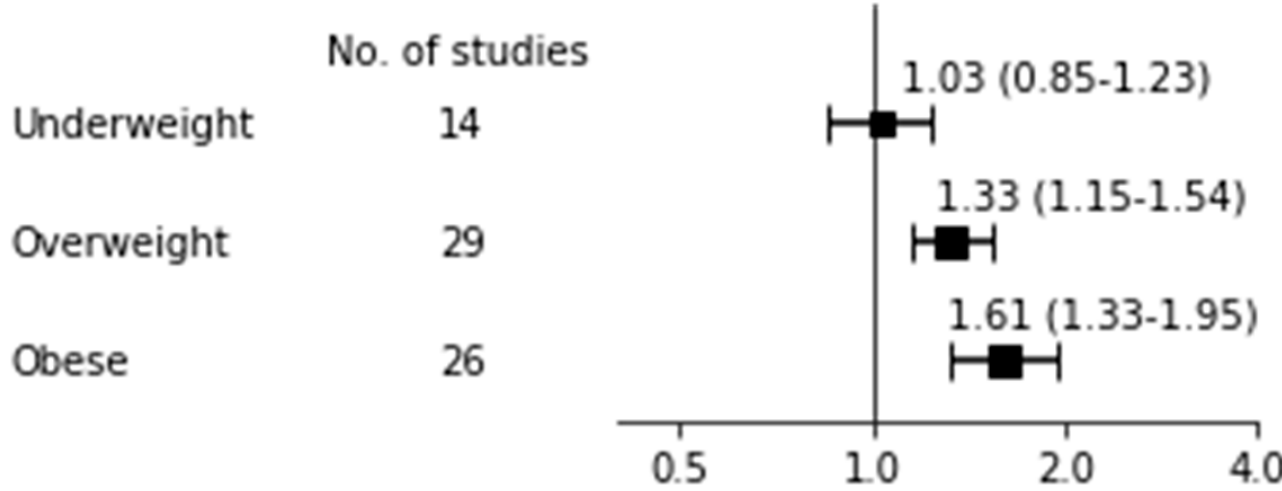

Figure 2. Breastfeeding non-initiation.

\subsection{Any Breastfeeding Cessation}

Table 3 shows the pooled risk for ABF cessation with $95 \% \mathrm{CI}$, heterogeneity, and publication bias and Figure 3 shows a forest plot for $\mathrm{ABF}$ cessation according to BMI categories. For studies that investigated underweight mothers, both the overall HR of four studies [70,76,102,115] $(1.28,95 \%$ CI: 0.71-2.31; $\mathrm{I}^{2}=95 \%, p$ for $\left.\mathrm{Q}=0.024, \mathrm{Tau}^{2}=0.32\right)$ and the OR of 10 studies $[69,71,73,92,96,97,101,103,109,116]$ (0.91, 95\% CI: $0.64-1.28$; $\mathrm{I}^{2}=86 \%, p$ for $\mathrm{Q}=0.002$, $\left.\mathrm{Tau}^{2}=0.19\right)$ were insignificant. For studies that investigated overweight mothers, overall HR was significant $\left(1.16,95 \%\right.$ CI: $1.07-1.25 ; I^{2}=23 \%$, $p$ for $\mathrm{Q}=0.913$, $\left.\mathrm{Tau}^{2}<0.01\right)$ among 10 studies $[67,70,76,81,82,84,102,110,111,115]$, indicating that overweight mothers had an increased risk of not continuing ABF compared to normal weight mothers. The significance was consistently observed in subgroup analyses of high quality scores $\geq 6\left(n=3[67,110,116]\right.$; HR, 1.14, 95\% CI: $1.03-1.26$; $\mathrm{I}^{2}=14 \%, p$ for $\mathrm{Q}=0.620$, $\left.\mathrm{Tau}^{2}<0.01\right)$, the six month assessment $\left(n=4[67,80,81,102]\right.$; HR 1.22, 95\% CI: $1.02-1.46 ; \mathrm{I}^{2}=29 \%, p$ for $\mathrm{Q}=0.440$, $\mathrm{Tau}^{2}<0.01$; data not shown), and white ethnicity ( $n=7[70,81,82,84,102,110,111]$; HR $1.19,95 \%$ CI: $1.09-1.29 ; \mathrm{I}^{2}=14 \%, p$ for $\mathrm{Q}=0.910$, $\mathrm{Tau}^{2}<0.01$; data not shown), confirming that overweight mothers had an increased risk of not continuing BF practices compared to normal weight mothers. On the other hand, the overall OR of being overweight on BF cessation was insignificant among 16 studies $[69,71-73,91-93,96,97,101,103,109-111,116,117]$ with substantial heterogeneity $\left(\mathrm{I}^{2} 89 \%, p\right.$ for $\mathrm{Q}$ $<0.001, \mathrm{Tau}^{2}=0.07$ ). For studies that investigated obese mothers, overall HR was significant (HR 1.43, 95\% CI: 1.22-1.69) among 10 studies [67,68,70,76,82,84,94,102,108,115] with substantial heterogeneity $\left(\mathrm{I}^{2} 68 \%, p\right.$ for $\mathrm{Q}=0.081$, Tau $\left.{ }^{2}=0.04\right)$. Such significance was also observed in the subgroup analyses including the six month assessment $\left(n=4\right.$ [67,82,94,102]; HR 1.66, 95\% CI: $1.19-2.31 ; \mathrm{I}^{2}=67 \%, p$ for $\mathrm{Q}$ $\left.=0.102, \mathrm{Tau}^{2}=0.08\right)$ and white ethnicity $(n=7$ [68,70,82,84,94,102,108]; HR 1.19, 95\% CI: $1.09-1.29$; $\mathrm{I}^{2}=14 \%, p$ for $\left.\mathrm{Q}=0.907, \mathrm{Tau}^{2}<0.01\right)$. The overall OR of being obese was significant (OR $1.47,95 \%$ CI: 1.16-1.86) among 18 studies [71-73,75,80,85,91-93,96,97,101,103,109-111,113,117] but heterogeneity was substantial $\left(\mathrm{I}^{2}=93 \%, p\right.$ for $\left.\mathrm{Q}<0.001, \mathrm{Tau}^{2}=0.20\right)$. The subgroup analyses showed that the pooled risk among seven studies with a higher quality score [71,73,96,97,101-111] (OR 1.71, 95\% CI: $1.34-2.18 ; \mathrm{I}^{2}=85 \%, p$ for $\mathrm{Q}<0.001$, Tau ${ }^{2}=0.06$ ), and the risk among 16 studies with white ethnicity [71,73,75,80,85,91-93,96,97,101,103,109-111,113] (OR 1.49, 95\% CI: 1.14-1.95; $I^{2}=94 \%$, $p$ for $\mathrm{Q}<0.001, \mathrm{Tau}^{2}=0.23$; data not shown) were both significant, but that among 12 studies in the six month assessment $[71,75,91,93,96,97,101,109-111,113,117]$ was insignificant (OR 1.37, 95\% CI: 0.99-1.90; $\mathrm{I}^{2}=96 \%, p$ for $\mathrm{Q}<0.001, \mathrm{Tau}^{2}=0.29$ ). 
Table 3. Pooled risk of underweight, overweight and obesity vs. normal weight on ABF cessation.

\begin{tabular}{|c|c|c|c|c|c|c|c|}
\hline Breastfeeding Cessation & $n$ & Pooled Risk (95\% CI) & Tau $^{2}$ & $I^{2}(\%)$ & $p$ for $Q$ & $\begin{array}{c}\text { Meta-Regression } \\
p \text {-Value }\end{array}$ & Begg's Test \\
\hline \multicolumn{8}{|c|}{ Underweight vs. normal weight } \\
\hline Hazard ratio & 4 & $1.28(0.71-2.31)$ & 0.317 & 95 & 0.024 & - & 0.013 \\
\hline $\begin{array}{c}\text { Odds ratio or risk ratio } \\
\text { Ouality score }\end{array}$ & 10 & $0.91(0.64-1.28)$ & 0.188 & 86 & 0.002 & - & 0.214 \\
\hline $6-$ & 7 & $0.87(0.54-1.39)$ & 0.299 & 93 & $<0.001$ & 0.915 & \\
\hline-5 & 3 & $0.92(0.58-1.44)$ & 0.005 & 2 & 0.854 & ref & \\
\hline \multicolumn{8}{|l|}{ Overweight vs. normal weight } \\
\hline \multicolumn{7}{|l|}{ Quality score } & 0.888 \\
\hline $6-$ & 3 & $1.14(1.03-1.26)$ & 0.001 & 14 & 0.620 & 0.626 & \\
\hline-5 & 7 & $1.18(1.07-1.31)$ & 0.004 & 19 & 0.836 & ref & \\
\hline \multicolumn{8}{|l|}{ Quality score } \\
\hline $6-$ & 9 & $1.28(1.07-1.53)$ & 0.048 & 86 & 0.001 & 0.125 & \\
\hline-5 & 7 & $1.04(0.79-1.38)$ & 0.099 & 81 & 0.001 & ref & \\
\hline \multicolumn{8}{|l|}{ Obese vs. normal weight } \\
\hline Hazard ratio & 10 & $1.43(1.22-1.69)$ & 0.044 & 68 & 0.081 & & 0.010 \\
\hline \multicolumn{8}{|l|}{ Quality score } \\
\hline $\begin{array}{l}6- \\
-5\end{array}$ & $\begin{array}{l}2 \\
8\end{array}$ & $\begin{array}{l}1.35(1.10-1.65) \\
1.48(1.21-1.81)\end{array}$ & $\begin{array}{l}<0.001 \\
0.056\end{array}$ & $\begin{array}{c}1 \\
73\end{array}$ & $\begin{array}{l}0.694 \\
0.033\end{array}$ & $\begin{array}{l}0.695 \\
\text { ref }\end{array}$ & \\
\hline Odds ratio or risk ratio & 18 & $1.47(1.16-1.86)$ & 0.200 & 93 & $<0.001$ & & 0.371 \\
\hline \multicolumn{8}{|l|}{ Quality score } \\
\hline $6-$ & 7 & $1.71(1.34-2.18)$ & 0.063 & 85 & $<0.001$ & 0.185 & \\
\hline-5 & 11 & $1.32(0.94-1.84)$ & 0.265 & 90 & $<0.001$ & ref & \\
\hline
\end{tabular}

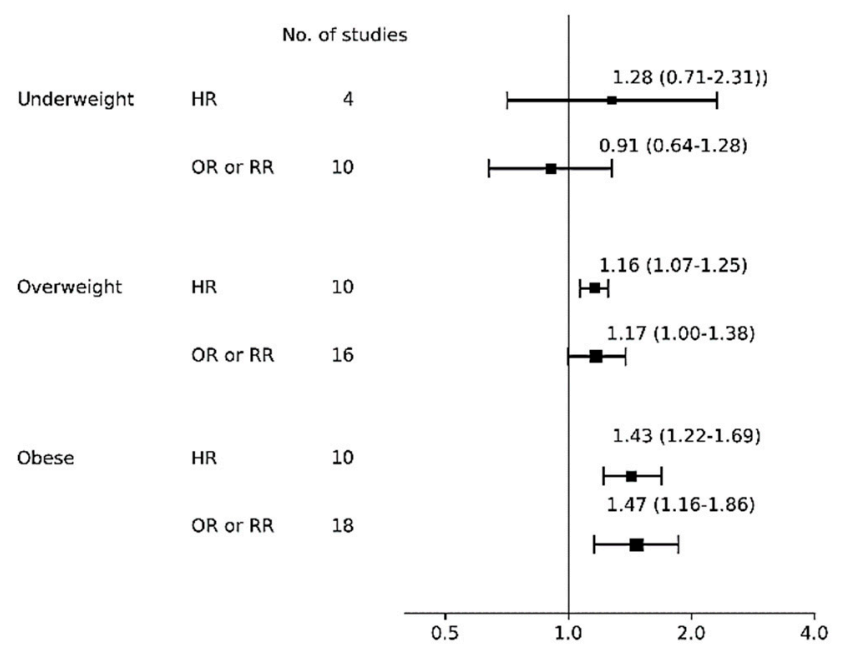

Figure 3. Forest plot for any breastfeeding cessation risk. Abbreviations: HR, hazard ratio; OR odds ratio; $R R$, risk ratio.

\subsection{Exclusive Breastfeeding Cessation}

Table 4 shows the pooled risk for not continuing EBF with 95\% CI, heterogeneity, and publication bias and Figure 4 shows a forest plot for EBF cessation risk according to BMI categories. For the effect of being underweight on EBF cessation, both the overall HR and OR did not reach significance. For the effect of being overweight, although the overall HR was insignificant, the overall OR reached significance for overweight women having an increased risk of not continuing EBF compared to normal weight women ( $n=12$ [71,90,95-97,100,103,104,106,114,115,117]; OR 1.37, 95\% CI: 1.10-1.72; I² $=96 \%, p$ for $\left.\mathrm{Q}<0.001, \mathrm{Tau}^{2}=0.13\right)$. As Begg's test was significant in overweight mothers, indicating publication bias, we additionally performed a trim-and-fill analysis by imputing missing studies and then observed insignificance (OR 1.16, 95\% CI: 0.97-1.37). The significance was observed across subgroup analyses of quality score $\geq 6\left(n=7\right.$ [71,90,95-97,104,115]; OR 1.15, 95\% CI: $1.08-1.24 ; \mathrm{I}^{2}=48 \%$, $p$ for $\mathrm{Q}=0.059$, $\left.\mathrm{Tau}^{2}<0.01\right)$ and white ethnicity $(n=9[71,95-97,100,103,104,106,114]$; OR $=1.51,95 \%$ CI: $1.13-2.02 ; \mathrm{I}^{2}=93 \%, p$ for $\mathrm{Q}<0.001, \mathrm{Tau}^{2}=0.16$; data not shown) but not in a subgroup analysis of 
six month timing of measurement $\left(n=5\right.$ [71,97,106,115,117]; OR 1.45, 95\% CI: $0.81-2.59 ; \mathrm{I}^{2}=98 \%, p$ for $\mathrm{Q}<0.001$, $\mathrm{Tau}^{2}<0.01$; data not shown). For the effect of obesity, the pooled risk was significant among seven studies $[67,70,87,93,94,102,119]$ with $\mathrm{HR}\left(1.45,95 \% \mathrm{CI}: 1.27-1.65 ; \mathrm{I}^{2}=44 \%, p\right.$ for $\mathrm{Q}=0.455$, Tau ${ }^{2}$ $=0.01)$ and insignificant among 11 studies $[71,90,95-97,100,103,104,115,117,118]$ with OR $(1.32,95 \%$ CI: $0.97-1.79 ; \mathrm{I}^{2}=96 \%, p$ for $\left.\mathrm{Q}<0.001, \mathrm{Tau}^{2}=0.21\right)$.

Table 4. Pooled risk of underweight, overweight and obesity vs. normal weight on EBF cessation.

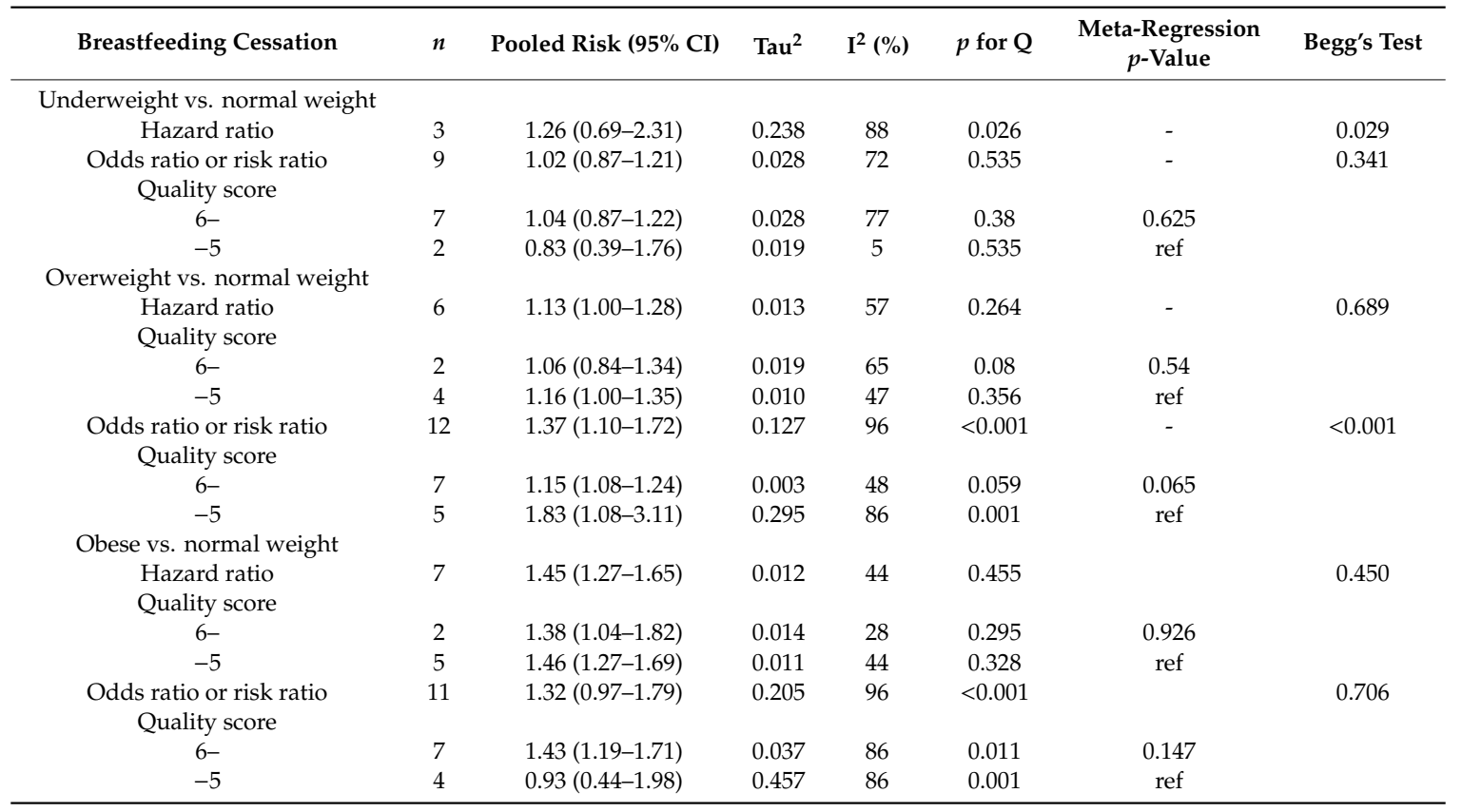

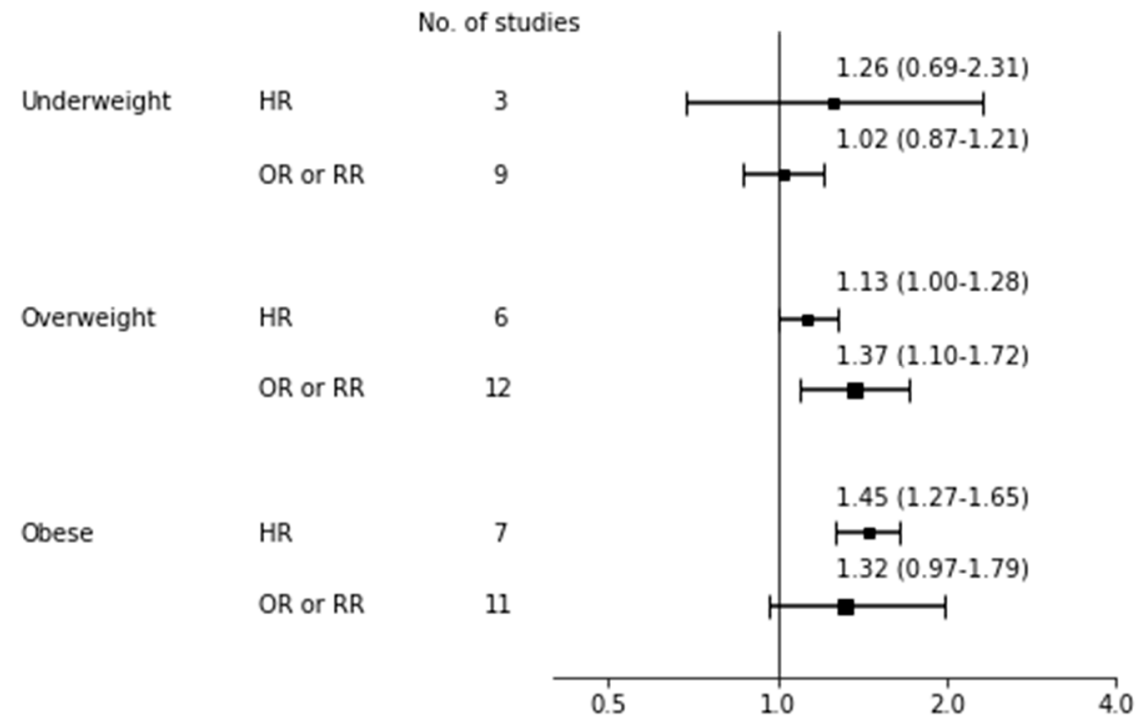

Figure 4. Forest plot for exclusive breastfeeding cessation risk. bbreviations: HR, hazard ratio; OR odds ratio; $R R$, risk ratio.

\section{Discussion}

This study investigated which maternal BMI categories are associated with BF non-initiation or cessation using a quantitative review. The effects of being underweight on BF non-initiation and cessation were all insignificant irrespective of the types of BF practice (i.e., ABF or EBF), or risk measurement (i.e., HR or OR). The pooled risks of non-initiation among those who were overweight and obese were both significant, although there was substantial heterogeneity observed. The pooled 
risk for $\mathrm{ABF}$ and $\mathrm{EBF}$ cessation in overweight and obese mothers was inconsistent with substantial heterogeneity. However, the hazard risks of ABF cessation in overweight mothers and EBF cessation in obese mothers were both significant with less heterogeneity (i.e., $\mathrm{I}^{2}=23 \%$ and $44 \%$, respectively). This quantitative review suggests that overweight and obese mothers may have an associated increased risk of BF non-initiation or cessation.

There are two previous meta-analysis studies [14,120] that investigated an association between maternal weight status and BF non-initiation and cessation which agree that obesity may be associated with an increased risk of BF non-initiation or cessation compared to normal weight mothers. However, their study hypotheses differ from ours; one study [120] not only included maternal weight status, but diet and supplement use as determinants of breastfeeding and complementary feeding; the other [14] investigated BF cessation associated with maternal BMI and gestational weight gain. The study conducted by Huang et al. [14] is similar to ours, but does not include cross-sectional studies, and thus includes only 30 studies. The exclusion of these studies may overlook the true association of interest because most studies that focused on breastfeeding initiation are cross-sectional studies. Hence, the advantages of our study are that, first, the numbers of studies included in our meta-analyses are much larger in our study than in Huang et al. We included 57 studies (initiation $n=29$, ABF cessation $n=31$, EBF cessation $n=20$ ) vs. 30 in Huang et al. (initiation $n=21$, ABF cessation $n=15$, EBF cessation $n=17$ ). Second, we also performed a comprehensive literature review including a large number of references (i.e., 130 in our study vs. 54 in Huang et al.). For example, our study encompassed an extended literature search not only limited to the studies included in the meta-analyses, but incorporating those studies that were excluded into Table S2. The description of studies excluded from the meta-analyses is important to reproduce a systematic review for a third person. Second, by performing pooled analyses according to odds or hazard ratios, the heterogeneity of some meta-analyses is less observed, which makes it clearer to present the true association of interest. The discrepancy is that Huang et al. reported underweight mothers to be less likely to initiate BF compared to normal weight mothers ( $n=14$, RR 1.28, 95\% CI:1.11-1.48). However, we did not observe such a negative impact among underweight women; both studies observed substantial heterogeneity across the studies included in the meta-analyses. In this regard, the effect of the mother being underweight on breastfeeding still requires further evidence.

There are several reasons for the substantial heterogeneity across the studies. First, there were only two studies awarded the maximum number of seven stars for quality, and the other studies were awarded six stars or fewer, which indicates the majority of studies included in these meta-analyses had, at least, a moderate risk of bias. The reason for the lower quality scores across studies includes the nondifferential misclassification or recall bias by self-reporting of maternal weight. In addition, the majority of the reviewed studies failed to provide follow-up rates that were long enough to assess breastfeeding duration. Indeed, few studies in which mothers continued breastfeeding up to six months (as recommended by the WHO and UNICEF) remained for analyses. Second, we excluded 10 studies [21-30] that provided continuous risk estimates because risk with a one unit increase in BMI is exponential, and thus difficult to interpret by combining the different sources of populations. Indeed, the 10 studies [21-30] vary across countries, BF types, and the length of the follow-up period. Nine studies [21-29] were reported from Western countries and three investigated initiation $[23,25,27]$. Among the seven studies [21-24,26,29,30] that assessed the risk of timing at six months after delivery, only two $[27,30]$ reported that the BF significantly decreased by a unit increase of BMI. Furthermore, we also attempted to collect mean BMI according to BF status in order to perform a quantitative assessment, but only two studies [25,29] provided such data. These two studies also reported the risks at different points in time (i.e., 18 months [25] and six months [29]) and thus we did not include these. Third, although we were able to assess the impact of being underweight on breastfeeding practice, because the studies in this area of research focus on obesity, the numbers of underweight studies included in the meta-analyses were few, especially for EBF cessation. Fourth, the WHO encourages EBF, especially in developing countries where maternal biological characteristics and socio-economic factors 
are significantly different from Western countries. In this regard, although there was no publication bias observed, the number of studies published from developing countries were still few, and thus the generalizability of the present meta-analyses may be limited.

Previously, several potential mechanisms have been reported. First, overweight or obese mothers have large heavy breasts, which may physiologically interfere with infant latching or adversely affect lactation [88]. Rasmussen and Kjolhed [121] demonstrated that excess adiposity in obese women contributes to dysregulation of the hypothalamic-pituitary-gonadal axis. In those women, the prolactin response to the baby's suckle is low with a delayed onset of milk production [122]. Some evidence from research in dairy cows and mice suggests that obesity in early life may negatively influence breast glandular development [123]. Obese mice also show a reduction in the milk proteins $\beta$-casein, whey acidic protein, and $\alpha$-lactalbumin, which are essential for milk production [124]. The breastmilk of normal weight women contains a higher concentration of medium-chain fatty acids; on the contrary, the milk of obese women is characterized by the presence of longer-chain fatty acids. These are more difficult to digest, especially in newborns with immature gastrointestinal systems, thus favoring supplementation with formula and/or solid food [125]. Another study showed that overweight women have a negative physiological pattern [126] influencing "maternal-fetal attachment". Overweight and obese women who are less likely to intend to breastfeed [127], had less confidence in sufficient milk supply and lower body shape satisfaction [128]. They often reported postpartum depression [129], and therefore bottle-feeding is more precocious. However, this association may be confounded by biological and social factors [130]. Finally, breastfeeding practice may be influenced by sociocultural factors. As our Begg's test indicates that publication bias may exist in our subgroup analyses, the majority of the reviewed studies were reported from developed countries that would be a great source of heterogeneity.

\section{Conclusions}

In summary, despite substantial heterogeneity across the reviewed studies, our quantitative review suggests that overweight and obese mothers are associated with breastfeeding non-initiation and cessation. Breastfeeding determinants are multifaceted, but among the relevant factors weight control is important to contribute to breastfeeding practice. This should be kept in mind for all relevant health care workers who are responsible for catering to women of reproductive age.

Supplementary Materials: The following are available online at http://www.mdpi.com/2072-6643/12/9/2684/s1, Table S1. Search Strategy, Table S2. Characteristics of excluded studies that investigated BMI as a continuous variable, Table S3. Newcastle-Ottawa Scale for the assessment of the methodological quality of included 57 studies.

Author Contributions: K.N. (Kyoko Nomura) and N.K. conceptualized and designed the study, ran individual literature searches, and critically reviewed and collected references for meta-analyses. K.N. (Kyoko Nomura) mainly carried out the analyses which was supervised by K.N. (Kengo Nagashima) for important intellectual content. K.N. (Kyoko Nomura) drafted the manuscript which was critically reviewed by all authors. S.M. and M.O. supervised data collection and reviewed and revised the manuscript. All authors approved the final manuscript as submitted and agree to be accountable for all aspects of the work. All authors have read and agreed to the published version of the manuscript.

Funding: All phrases of this study were supported by grant from the Ministry of Sports, Culture, and Science (the Grants-in-Aid for Scientific-Research Program No. 16H052621).

Acknowledgments: We thank Masayuki Shiraishi who belongs to Osaka University for his assistance with the literature search and Ayumi Tanabe and Saki Horie for data extraction from the literature. In addition, we thank Murray Mittleman and Elizabeth Mostofsky, from the Department of Epidemiology Harvard T.H. Chan School of Public Health, for their valuable comments.

Conflicts of Interest: The authors declare no conflict of interest.

\section{References}

1. Dogaru, C.M.; Nyffenegger, D.; Pescatore, A.M.; Spycher, B.D.; Kuehni, C.E. Breastfeeding and Childhood Asthma: Systematic Review and Meta-Analysis. Am. J. Epidemiol. 2014, 179, 1153-1167. [CrossRef] [PubMed] 
2. Amitay, E.L.; Keinan-Boker, L. Breastfeeding and Childhood Leukemia Incidence: A meta-analysis and systematic review. JAMA Pediatr. 2015, 169, e151025. [CrossRef] [PubMed]

3. Horwood, L.J.; Fergusson, D.M. Breastfeeding and later cognitive and academic outcomes. Pediatrics 1998, 101, e9. [CrossRef] [PubMed]

4. Yan, J.; Liu, L.; Zhu, Y.; Huang, G.; Wang, P.P. The association between breastfeeding and childhood obesity: A meta-analysis. BMC Public Health 2014, 14, 1267. [CrossRef]

5. Walker, C.L.F.; Rudan, I.; Liu, L.; Nair, H.; Theodoratou, E.; Bhutta, Z.A.; O’brien, K.L.; Campbell, H.; Black, R.E. Global burden of childhood pneumonia and diarrhoea. Lancet 2013, 381, 1405-1416. [CrossRef]

6. Beral, V.; Bull, D.; Doll, R.; Peto, R.; Reeves, G. Breast cancer and breastfeeding: Collaborative reanalysis of individual data from 47 epidemiological studies in 30 countries, including 50302 women with breast cancer and 96973 women without the disease. Lancet 2002, 360, 187-195.

7. Aune, D.; Norat, T.; Romundstad, P.R.; Vatten, L. Breastfeeding and the maternal risk of type 2 diabetes: A systematic review and dose-response meta-analysis of cohort studies. Nutr. Metab. Cardiovasc. Dis. 2014, 24, 107-115. [CrossRef]

8. Chowdhury, R.; Sinha, B.; Sankar, M.J.; Taneja, S.; Bhandari, N.; Rollins, N.; Bahl, R.; Martines, J. Breastfeeding and maternal health outcomes: A systematic review and meta-analysis. Acta Paediatr. 2015, 104, 96-113. [CrossRef]

9. Victora, C.G.; Bahl, R.; Barros, A.J.D.; França, G.V.A.; Horton, S.; Krasevec, J.; Murch, S.; Sankar, M.J.; Walker, N.; Rollins, N.C. Breastfeeding in the 21st century: Epidemiology, mechanisms, and lifelong effect. Lancet 2016, 387, 475-490. [CrossRef]

10. McAndrew, F.; Thompson, J.; Fellows, L.; Large, A.; Speed, M.R.M. Infant Feeding Survey 2010; Health and Social Care Information Centre: Leeds, UK, 2012.

11. Centers for DiseasePrevention (CDC). Breastfeeding among U.S. Children born 2001-2011. Atlanta. 2014. Report No.: GA2014. Available online: http://www.webcitation.org/6ZVxfUTT6 (accessed on 1 September 2020).

12. Britt Lande, A.H. Amming og Spedbarns Kosthold: Landsomfattende Undersøkelse 2013; Helsedirektoratet: Oslo, Norway, 2014.

13. Breast-Feeding and Smoking Habits among Parents of Infants Born in 2010; Official Statistics of Sweden: Stockholm, Sweden, 2012.

14. Huang, Y.; Ouyang, Y.-Q.; Redding, S.R. Maternal Prepregnancy Body Mass Index, Gestational Weight Gain, and Cessation of Breastfeeding: A Systematic Review and Meta-Analysis. Breastfeed. Med. 2019, 14, 366-374. [CrossRef]

15. Murai, U.; Nomura, K.; Kido, M.; Takeuchi, T.; Sugimoto, M.; Rahman, M. Pre-pregnancy body mass index as a predictor of low birth weight infants in Japan. Asia Pac. J. Clin. Nutr. 2017, 26, 434-437. [PubMed]

16. Nomura, K.; Kido, M.; Tanabe, A.; Ando, K. Prepregnancy obesity as a risk factor for exclusive breastfeeding initiation in Japanese women. Nutrition 2019, 62, 93-99. [CrossRef] [PubMed]

17. Shamseer, L.; Moher, D.; Clarke, M.; Ghersi, D.; Liberati, A.; Petticrew, M.; Shekelle, P.; Stewart, L.A. Preferred reporting items for systematic review and meta-analysis protocols (PRISMA-P) 2015: Elaboration and explanation. BMJ 2015, 350, g7647. [CrossRef] [PubMed]

18. WHO. WHO|Global Database on Body Mass Index (BMI); World Health Organization: Geneva, Switzerland, 2010.

19. World Health Organization; Regional Office for the Western Pacific. The Asia-Pacific Perspective: Redefining Obesity and its Treatment; Health Communications Australia: Sydney, Australia, 2000; Available online: http://iris.wpro.who.int/handle/10665.1/5379 (accessed on 1 September 2020).

20. Part, I. Weight Gain. Part II. Nutrient Supplements. In Nutrition during Pregnancy; National Academies Press: Washington, WA, USA, 1990; ISBN 0-309-04138-4.

21. Giovannini, M.; Riva, E.; Banderali, G.; Scaglioni, S.; Veehof, S.H.; Sala, M.; Radaelli, G.; Agostoni, C. Feeding practices of infants through the first year of life in Italy. Acta Paediatr. 2004, 93, 492-497. [CrossRef] [PubMed]

22. Theofilogiannakou, M.; Skouroliakou, M.; Gounaris, A.; Panagiotakos, D.; Markantonis, S.L. Breast-feeding in Athens, Greece. J. Pediatr. Gastroenterol. Nutr. 2006, 43, 379-384. [CrossRef] [PubMed]

23. Flower, K.B.; Willoughby, M.T.; Cadigan, R.J.; Perrin, E.M.; Randolph, G. The Family Life Project Investigative Team Understanding Breastfeeding Initiation and Continuation in Rural Communities: A Combined Qualitative/Quantitative Approach. Matern. Child Health J. 2007, 12, 402-414. [CrossRef] [PubMed] 
24. Jessri, M.; Farmer, A.P.; Maximova, K.; Willows, N.D.; Bell, R.C. Predictors of exclusive breastfeeding: Observations from the Alberta pregnancy outcomes and nutrition (APrON) study. BMC Pediatr. 2013, 13, 77. [CrossRef]

25. Vassilaki, M.; Chatzi, L.; Bagkeris, E.; Papadopoulou, E.; Karachaliou, M.; Koutis, A.; Philalithis, A.; Kogevinas, M. Smoking and caesarean deliveries: Major negative predictors for breastfeeding in the mother-child cohort in Crete, Greece (Rhea study). Matern. Child Nutr. 2012, 10, 335-346. [CrossRef]

26. Tavoulari, E.-F.; Benetou, V.; Vlastarakos, P.V.; Kreatsas, G.; Linos, A. Immigrant Status as Important Determinant of Breastfeeding Practice in Southern Europe. Central Eur. J. Public Health 2015, 23, 39-44. [CrossRef]

27. Tavoulari, E.-F.; Benetou, V.; Vlastarakos, P.V.; Andriopoulou, E.; Kreatsas, G.; Linos, A. Factors affecting breast-feeding initiation in Greece: What is important? Midwifery 2015, 31, 323-331. [CrossRef]

28. Zanardo, V.; Cavallin, F.; Guerrini, P.; Bertin, M.; Straface, G. Prepregnancy Body Mass Index Shift Across Gestation: An Association with Breastfeeding Practices? Breastfeed. Med. 2017, 12, 615-620. [CrossRef] [PubMed]

29. Zimmerman, E.; Rodgers, R.F.; O'Flynn, J.; Bourdeau, A. Weight-Related Concerns as Barriers to Exclusive Breastfeeding at 6 Months. J. Hum. Lact. 2019, 35, 284-291. [CrossRef] [PubMed]

30. Mangel, L.; Mimouni, F.B.; Mandel, D.; Mordechaev, N.; Marom, R. Breastfeeding Difficulties, Breastfeeding Duration, Maternal Body Mass Index, and Breast Anatomy: Are They Related? Breastfeed. Med. 2019, 14, 342-346. [CrossRef]

31. World Health Organization. WHO|Indicators for Assessing Infant and Young Child Feeding Practices; World Health Organization: Geneva, Switzerland, 2014.

32. Wells, G.A.; Shea, B.; O'Connell, D.; Peterson, J.; Welch, V.; Losos, M.; Tugwell, P. The Newcastle-Ottawa Scale (NOS) for Assessing the Quality of Nonrandomised Studies in Meta-Analyses. 2011. Available online: http://www.ohri.ca/programs/clinical_epidemiology/oxford.asp (accessed on 5 March 2020).

33. Veroniki, A.A.; Jackson, D.; Bender, R.; Kuss, O.; Langan, D.; Higgins, J.P.T.; Knapp, G.; Salanti, G. Methods to calculate uncertainty in the estimated overall effect size from a random-effects meta-analysis. Res. Synth. Methods 2018, 10, 23-43. [CrossRef] [PubMed]

34. Borenstein, M.; Hedges, L.V.; Higgins, J.P.T.; Rothstein, H.R. Introduction to Meta-Analysis. Introd. Meta-Anal. 2009, 27, 1-421. [CrossRef]

35. Higgins, J.P.T. Commentary: Heterogeneity in meta-analysis should be expected and appropriately quantified. Int. J. Epidemiol. 2008, 37, 1158-1160. [CrossRef]

36. Higgins, J.P.T.; Thompson, S.G.; Deeks, J.J.; Altman, D.G. Measuring inconsistency in meta-analyses. BMJ 2003, 327, 557-560. [CrossRef]

37. Martinez-Brockman, J.L.; Chapman, D.J.; Pérez-Escamilla, R. Prepregnancy Obesity Class Is a Risk Factor for Failure to Exclusively Breastfeed at Hospital Discharge among Latinas. J. Hum. Lact. 2016, 32, 258-268. [CrossRef]

38. Nohr, E.A.; Timpson, N.J.; Andersen, C.S.; Smith, G.D.; Olsen, J.; Sørensen, T.I.A. Severe Obesity in Young Women and Reproductive Health: The Danish National Birth Cohort. PLoS ONE 2009, 4, e8444. [CrossRef]

39. Cordero, L.; Valentine, C.J.; Samuels, P.; Giannone, P.J.; Nankervis, C.A. Breastfeeding in Women with Severe Preeclampsia. Breastfeed. Med. 2012, 7, 457-463. [CrossRef]

40. Cox, K.; Giglia, R.C.; Zhao, Y.; Binns, C.W. Factors Associated with Exclusive Breastfeeding at Hospital Discharge in Rural Western Australia. J. Hum. Lact. 2014, 30, 488-497. [CrossRef] [PubMed]

41. Cordero, L.; Oza-Frank, R.; Landon, M.B.; Nankervis, C.A. Breastfeeding Initiation Among Macrosomic Infants Born to Obese Nondiabetic Mothers. Breastfeed. Med. 2015, 10, 239-245. [CrossRef] [PubMed]

42. Kachoria, R.; Oza-Frank, R. Differences in Breastfeeding Initiation by Maternal Diabetes Status and Race, Ohio 2006-2011. Matern. Child Health J. 2014, 18, 2226-2235. [CrossRef] [PubMed]

43. Matias, S.; Dewey, K.G.; Quesenberry, C.P.; Gunderson, E.P. Maternal prepregnancy obesity and insulin treatment during pregnancy are independently associated with delayed lactogenesis in women with recent gestational diabetes mellitus. Am. J. Clin. Nutr. 2013, 99, 115-121. [CrossRef] [PubMed]

44. Soltani, H.; Arden, M. Factors Associated With Breastfeeding Up to 6 Months Postpartum in Mothers with Diabetes. J. Obstet. Gynecol. Neonatal Nurs. 2009, 38, 586-594. [CrossRef]

45. Almqvist-Tangen, G.; Bergman, S.; Dahlgren, J.; Roswall, J.; Alm, B. Factors associated with discontinuation of breastfeeding before 1 month of age. Acta Paediatr. 2012, 101, 55-60. [CrossRef] [PubMed] 
46. Al-Sahab, B.; Tamim, H.; Mumtaz, G.; Khawaja, M.; Khogali, M.; Afifi, R.A.; Nassif, Y.; Yunis, K.A. Predictors of breast-feeding in a developing country: Results of a prospective cohort study. Public Health Nutr. 2008, 11, 1350-1356. [CrossRef]

47. Boudet-Berquier, J.; Salanave, B.; Desenclos, J.-C.; Castetbon, K. Association between maternal prepregnancy obesity and breastfeeding duration: Data from a nationwide prospective birth cohort. Matern. Child Nutr. 2017, 14, 1-12. [CrossRef]

48. Chapman, D.J.; Pérez-Escamilla, R. Does delayed perception of the onset of lactation shorten breastfeeding duration? J. Hum. Lact. 1999, 15, 107-111. [CrossRef]

49. Demétrio, F.; de Pinto, E.J.; Oliveira Assis, A.M. Factors associated with early breastfeeding cessation: A birth cohort study in two municipalities in the Recôncavo region, Bahia State, Brazil. Cad. Saude Publica 2012, 28, 641-654. [CrossRef]

50. Giovannini, M.; Radaelli, G.; Riva, E.; Banderali, G. Low Prepregnant Body Mass Index and Breastfeeding Practices. J. Hum. Lact. 2007, 23, 44-51. [CrossRef] [PubMed]

51. Guelinckx, I.; Devlieger, R.; Bogaerts, A.; Pauwels, S.; VanSant, G. The effect of pre-pregnancy BMI on intention, initiation and duration of breast-feeding. Public Health Nutr. 2012, 15, 840-848. [CrossRef] [PubMed]

52. Holbrook, K.; White, M.C.; Heyman, M.B.; Wojcicki, J.M. Maternal sociodemographic characteristics and the use of the Iowa Infant Attitude Feeding Scale to describe breastfeeding initiation and duration in a population of urban, Latina mothers: A prospective cohort study. Int. Breastfeed. J. 2013, 8, 7. [CrossRef] [PubMed]

53. Panaviene, J.; Zakharchenko, L.; Olteanu, D.; Cullen, M.; El-Khuffash, A. Factors Contributing to Non-Exclusive Breastfeeding in Primigravid Mothers. Ir. Med. J. 2019, 112, 1003. [PubMed]

54. Locke, R.O.; Paul, D.; DiMatteo, D. Breastfeeding continuation factors in a cohort of Delaware mothers. Del. Med. J. 2006, 78, 295-300. [PubMed]

55. Mehta, U.J.; Siega-Riz, A.M.; Herring, A.H.; Adair, L.S.; Bentley, M.E. Pregravid body mass index, psychological factors during pregnancy and breastfeeding duration: Is there a link? Matern. Child Nutr. 2012, 8, 423-433. [CrossRef]

56. Gigante, D.P.; Victora, C.G.; Barros, F.C. Relationship between maternal nutrition and duration of breastfeeding in a birth cohort in Southern Brazil. Rev. Saude Publica 2000, 34, 259-265. [CrossRef]

57. Quandt, S.A. Biological and behavioral predictors of exclusive breastfeeding duration. Med. Anthr. 1985, 9 , 139-151. [CrossRef]

58. Ramakrishnan, R.; Oberg, C.N.; Kirby, R.S. The Association between Maternal Perception of Obstetric and Pediatric Care Providers' Attitudes and Exclusive Breastfeeding Outcomes. J. Hum. Lact. 2014, 30, 80-87. [CrossRef]

59. Rutishauser, I.H.; Carlin, J.B. Body mass index and duration of breast feeding: A survival analysis during the first six months of life. J. Epidemiol. Community Health 1992, 46, 559-565. [CrossRef]

60. Scott, J.A.; Binns, C.W.; Graham, K.I.; Oddy, W.H. Temporal Changes in the Determinants of Breastfeeding Initiation. Birth 2006, 33, 37-45. [CrossRef] [PubMed]

61. Sellen, D.W.; Thompson, A.L.; Hruschka, D.J.; Stein, A.; Martorell, R. Early Determinants of Non-Exclusive Breastfeeding among Guatemalan Infants; Springer: Boston, MA, USA, 2004; pp. 299-301.

62. Susiloretni, K.A.; Hadi, H.; Blakstad, M.; Smith, E.R.; Shankar, A.H. Does exclusive breastfeeding relate to the longer duration of breastfeeding? A prospective cohort study. Midwifery 2019, 69, 163-171. [CrossRef] [PubMed]

63. Tönz, O.; Schwaninger, U.; Holzherr, E.; Schafroth, M. Infant nutrition in Switzerland 1978. A prospective study on the nutritional habits during the first 6 months of life. I. Natural nutrition: Breast feeding. Schweiz. Med. Wochenschr. 1980, 110, 937-947. [PubMed]

64. Vingraite, J.; Bartkeviciute, R.; Michaelsen, K.F. A cohort study of term infants from Vilnius, Lithuania: Feeding patterns. Acta Paediatr. 2007, 93, 1349-1355. [CrossRef]

65. Hilson, J.A.; Rasmussen, K.M.; Kjolhede, C.L. Maternal obesity and breast-feeding success in a rural population of white women. Am. J. Clin. Nutr. 1997, 66, 1371-1378. [CrossRef]

66. Sebire, N.; Jolly, M.; Harris, J.; Wadsworth, J.; Joffe, M.; Beard, R.W.; Regan, L.; Robinson, S.; Sebire, N.J. Maternal obesity and pregnancy outcome: A study of 287213 pregnancies in London. Int. J. Obes. 2001, 25, 1175-1182. [CrossRef] 
67. Kugyelka, J.G.; Rasmussen, K.M.; Frongillo, E.A. Maternal Obesity is Negatively Associated with Breastfeeding Success among Hispanic but Not Black Women. J. Nutr. 2004, 134, 1746-1753. [CrossRef]

68. Hilson, J.A.; Rasmussen, K.M.; Kjolhede, C.L. High Prepregnant Body Mass Index is Associated With Poor Lactation Outcomes Among White, Rural Women Independent of Psychosocial and Demographic Correlates. J. Hum. Lact. 2004, 20, 18-29. [CrossRef]

69. Grjibovski, A.M.; Yngve, A.; Bygren, L.O.; Sjöström, M. Socio-demographic determinants of initiation and duration of breastfeeding in northwest Russia. Acta Paediatr. 2005, 94, 588-594. [CrossRef]

70. Hilson, J.A.; Rasmussen, K.M.; Kjolhede, C.L. Excessive Weight Gain during Pregnancy Is Associated with Earlier Termination of Breast-Feeding among White Women. J. Nutr. 2006, 136, 140-146. [CrossRef]

71. Baker, J.L.; Michaelsen, K.F.; Sørensen, T.I.; Rasmussen, K.M. High prepregnant body mass index is associated with early termination of full and any breastfeeding in Danish women 1-3. Am. J. Clin. Nutr. 2007, 86, 404-411. [CrossRef] [PubMed]

72. Jain, N.J.; Denk, C.E.; Kruse, L.K.; Dandolu, V. Maternal Obesity: Can Pregnancy Weight Gain Modify Risk of Selected Adverse Pregnancy Outcomes? Am. J. Perinatol. 2007, 24, 291-298. [CrossRef] [PubMed]

73. Manios, Y.; Grammatikaki, E.; Kondaki, K.; Ioannou, E.; Anastasiadou, A.; Birbilis, M. The effect of maternal obesity on initiation and duration of breast-feeding in Greece: The GENESIS study. Public Heal. Nutr. 2009, 12, 517-524. [CrossRef] [PubMed]

74. Fairlie, T.G.; Gillman, M.W.; Rich-Edwards, J. High Pregnancy-Related Anxiety and Prenatal Depressive Symptoms as Predictors of Intention to Breastfeed and Breastfeeding Initiation. J. Women's Health 2009, 18, 945-953. [CrossRef] [PubMed]

75. Kehler, H.L.; Chaput, K.H.; Tough, S.C. Risk Factors for Cessation of Breastfeeding Prior to Six Months Postpartum among a Community Sample of Women in Calgary, Alberta. Can. J. Public Health 2009, 100, 376-380. [CrossRef]

76. Liu, J.; Smith, M.G.; Dobre, M.A.; Ferguson, J.E. Maternal Obesity and Breast-feeding Practices among White and Black Women. Obesity 2010, 18, 175-182. [CrossRef]

77. Kitsantas, P.; Pawloski, L.R. Maternal obesity, health status during pregnancy, and breastfeeding initiation and duration. J. Matern. Neonatal Med. 2010, 23, 135-141. [CrossRef]

78. Mehta, U.J.; Siega-Riz, A.M.; Herring, A.H.; Adair, L.S.; Bentley, M.E. Maternal Obesity, Psychological Factors, and Breastfeeding Initiation. Breastfeed. Med. 2011, 6, 369-376. [CrossRef]

79. Tenfelde, S.; Finnegan, L.; Hill, P.D. Predictors of Breastfeeding Exclusivity in a WIC Sample. J. Obstet. Gynecol. Neonatal Nurs. 2011, 40, 179-189. [CrossRef]

80. Leonard, S.A.; Labiner-Wolfe, J.; Geraghty, S.R.; Rasmussen, K.M. Associations between high prepregnancy body mass index, breast-milk expression, and breast-milk production and feeding. Am. J. Clin. Nutr. 2011, 93, 556-563. [CrossRef]

81. Hauff, L.E.; Demerath, E.W. Body image concerns and reduced breastfeeding duration in primiparous overweight and obese women. Am. J. Hum. Boil. 2012, 24, 339-349. [CrossRef] [PubMed]

82. Bartok, C.J.; Schaefer, E.W.; Beiler, J.S.; Paul, I.M. Role of Body Mass Index and Gestational Weight Gain in Breastfeeding Outcomes. Breastfeed. Med. 2012, 7, 448-456. [CrossRef] [PubMed]

83. Thompson, L.A.; Zhang, S.; Black, E.W.; Das, R.; Ryngaert, M.; Sullivan, S.; Roth, J. The Association of Maternal Pre-pregnancy Body Mass Index with Breastfeeding Initiation. Matern. Child Health J. 2013, 17, 1842-1851. [CrossRef] [PubMed]

84. Kronborg, H.; Vaeth, M.; Rasmussen, K.M. Obesity and early cessation of breastfeeding in Denmark. Eur. J. Public Health 2012, 23, 316-322. [CrossRef]

85. Branger, B.; Dinot-Mariau, L.; Lemoine, N.; Godon, N.; Merot, E.; Brehu, S.; Winer, N.; Brossier, J.-P. Durée d'allaitement maternel et facteurs de risques d'arrêt d'allaitement: Évaluation dans 15 maternités du Réseau de santé en périnatalité des Pays de la Loire. Archives de Pédiatrie 2012, 19, 1164-1176. [CrossRef]

86. Sipsma, H.L.; Magriples, U.; Divney, A.A.; Gordon, D.; Gabzdyl, E.; Kershaw, T. Breastfeeding behavior among adolescents: Initiation, duration, and exclusivity. J. Adolesc. Health 2013, 53, 394-400. [CrossRef]

87. Brown, C.R.; Dodds, L.; Attenborough, R.; Bryanton, J.; Rose, A.E.; Flowerdew, G.; Langille, D.; Lauzon, L.; Semenic, S. Rates and determinants of exclusive breastfeeding in first 6 months among women in Nova Scotia: A population-based cohort study. CMAJ Open 2013, 1, E9-E17. [CrossRef] 
88. Gubler, T.; Krähenmann, F.; Roos, M.; Zimmermann, R.; Ochsenbein-Kölble, N. Determinants of successful breastfeeding initiation in healthy term singletons: A Swiss university hospital observational study. J. Perinat. Med. 2013, 41, 331-339. [CrossRef]

89. Visram, H.; Finkelstein, S.A.; Feig, D.; Walker, M.; Yasseen, A.; Tu, X.; Keely, E. Breastfeeding intention and early post-partum practices among overweight and obese women in Ontario: A selective population-based cohort study. J. Matern. Neonatal Med. 2013, 26, 611-615. [CrossRef]

90. Hayes, D.K.; Mitchell, K.M.; Donohoe-Mather, C.; Zaha, R.L.; Melcher, C.; Fuddy, L.J. Predictors of exclusive breastfeeding at least 8 weeks among Asian and Native Hawaiian or other Pacific Islander race subgroups in Hawaii, 2004-2008. Matern. Child Health J. 2014, 18, 1215-1223. [CrossRef]

91. Vurbic, I.; Higgins, S.T.; McDonough, S.R.; Skelly, J.M.; Bernstein, I.M. Maternal Body Mass Index Moderates the Influence of Smoking Cessation on Breast Feeding. Nicotine Tob. Res. 2014, 16, 527-535. [CrossRef] [PubMed]

92. Stuebe, A.M.; Horton, B.J.; Chetwynd, E.; Watkins, S.; Grewen, K.; Meltzer-Brody, S. Prevalence and Risk Factors for Early, Undesired Weaning Attributed to Lactation Dysfunction. J. Women's Health 2014, 23, 404-412. [CrossRef] [PubMed]

93. Hauff, L.E.; Leonard, S.A.; Rasmussen, K.M. Associations of maternal obesity and psychosocial factors with breastfeeding intention, initiation, and duration. Am. J. Clin. Nutr. 2014, 99, 524-534. [CrossRef] [PubMed]

94. Cox, K.; Binns, C.W.; Giglia, R.C. Predictors of breastfeeding duration for rural women in a high-income country: Evidence from a cohort study. Acta Paediatr. 2015, 104, e350-e359. [CrossRef] [PubMed]

95. Perrine, C.G.; Scanlon, K.S.; Li, R.; Odom, E.; Grummer-Strawn, L.M. Baby-Friendly hospital practices and meeting exclusive breastfeeding intention. Pediatrics 2012, 130, 54-60. [CrossRef] [PubMed]

96. Smith, H.A.; Hourihane, J.O.; Kenny, L.C.; Kiely, M.E.; Murray, D.M.; Leahy-Warren, P. Early life factors associated with the exclusivity and duration of breast feeding in an Irish birth cohort study. Midwifery 2015, 31, 904-911. [CrossRef]

97. Winkvist, A.; Brantsæter, A.L.; Brandhagen, M.; Haugen, M.; Meltzer, H.M.; Lissner, L. Maternal Prepregnant Body Mass Index and Gestational Weight Gain Are Associated with Initiation and Duration of Breastfeeding among Norwegian Mothers. J. Nutr. 2015, 145, 1263-1270. [CrossRef]

98. Verret-Chalifour, J.; Giguère, Y.; Forest, J.-C.; Croteau, J.; Zhang, P.; Marc, I. Breastfeeding Initiation: Impact of Obesity in a Large Canadian Perinatal Cohort Study. PLoS ONE 2015, 10, e0117512. [CrossRef]

99. Masho, S.W.; Cha, S.; Morris, M.R. Prepregnancy Obesity and Breastfeeding Noninitiation in the United States: An Examination of Racial and Ethnic Differences. Breastfeed. Med. 2015, 10, 253-262. [CrossRef]

100. Lindau, J.F.; Mastroeni, S.; Gaddini, A.; Di Lallo, D.; Nastro, P.F.; Patane', M.; Girardi, P.; Fortes, C. Determinants of exclusive breastfeeding cessation: Identifying an "at risk population" for special support. Eur. J. Nucl. Med. Mol. Imaging 2015, 174, 533-540. [CrossRef]

101. Kair, L.R.; Colaizy, T.T. Breastfeeding Continuation among Late Preterm Infants: Barriers, Facilitators, and Any Association with NICU Admission? Hosp. Pediatr. 2016, 6, 261-268. [CrossRef] [PubMed]

102. Logan, C.; Zittel, T.; Striebel, S.; Reister, F.; Brenner, H.; Rothenbacher, D.; Genuneit, J. Changing Societal and Lifestyle Factors and Breastfeeding Patterns Over Time. Pediatrics 2016, 137. [CrossRef] [PubMed]

103. Bjørset, V.K.; Helle, C.; Hillesund, E.R.; Øverby, N.C. Socio-economic status and maternal BMI are associated with duration of breast-feeding of Norwegian infants. Public Heal. Nutr. 2018, 21, 1465-1473. [CrossRef] [PubMed]

104. Haile, Z.T.; Elmasry, M.; Chavan, B.; Chertok, I.R.A. Association between Type of Health Professional at Birth and Exclusive Breastfeeding. J. Midwifery Women's Health 2017, 62, 562-571. [CrossRef] [PubMed]

105. Wallenborn, J.T.; Graves, W.C.; Masho, S.W. Breastfeeding Initiation in Mothers with Repeat Cesarean Section: The Impact of Marital Status. Breastfeed. Med. 2017, 12, 227-232. [CrossRef] [PubMed]

106. Marshall, N.E.; Lau, B.; Purnell, J.Q.; Thornburg, K.L. Impact of maternal obesity and breastfeeding intention on lactation intensity and duration. Matern. Child Nutr. 2019, 15, e12732. [CrossRef] [PubMed]

107. Ramji, N.; Challa, S.; Murphy, P.A.; Quinlan, J.; Crane, J.M.G. A comparison of breastfeeding rates by obesity class. J. Matern. Neonatal Med. 2018, 31, 3021-3026. [CrossRef]

108. Donath, S.; Amir, L.H. Does maternal obesity adversely affect breastfeeding initiation and duration? J. Paediatr. Child Health 2000, 36, 482-486. [CrossRef]

109. Forster, D.A.; McLachlan, H.; Lumley, J. Factors associated with breastfeeding at six months postpartum in a group of Australian women. Int. Breastfeed. J. 2006, 1, 18. [CrossRef] 
110. Oddy, W.H.; Li, J.; Landsborough, L.; Kendall, G.E.; Henderson, S.; Downie, J. The association of maternal overweight and obesity with breastfeeding duration. J. Pediatr. 2006, 149, 185-191. [CrossRef]

111. Donath, S.; Amir, L.H. Maternal obesity and initiation and duration of breastfeeding: Data from the longitudinal study of Australian children. Matern. Child Nutr. 2008, 4, 163-170. [CrossRef] [PubMed]

112. Biro, M.A.; Sutherland, G.A.; Yelland, J.; Hardy, P.; Brown, S.J. In-Hospital Formula Supplementation of Breastfed Babies: A Population-Based Survey. Birth 2011, 38, 302-310. [CrossRef] [PubMed]

113. Forster, D.A.; Johns, H.M.; McLachlan, H.L.; Moorhead, A.M.; McEgan, K.M.; Amir, L.H. Feeding infants directly at the breast during the postpartum hospital stay is associated with increased breastfeeding at 6 months postpartum: A prospective cohort study. BMJ Open 2015, 5, 4-9. [CrossRef] [PubMed]

114. De Jersey, S.J.; Mallan, K.M.; Forster, J.; Daniels, L.A. A prospective study of breastfeeding intentions of healthy weight and overweight women as predictors of breastfeeding outcomes. Midwifery 2017, 53, $20-27$. [CrossRef] [PubMed]

115. Tao, X.-Y.; Huang, K.; Yan, S.-Q.; Zuo, A.-Z.; Tao, R.-W.; Cao, H.; Gu, C.-L.; Tao, F.-B. Pre-pregnancy BMI, gestational weight gain and breast-feeding: A cohort study in China. Public Health Nutr. 2017, 20, 1001-1008. [CrossRef]

116. Zhu, P.; Hao, J.; Jiang, X.; Huang, K.; Tao, F. New Insight into Onset of Lactation: Mediating the Negative Effect of Multiple Perinatal Biopsychosocial Stress on Breastfeeding Duration. Breastfeed. Med. 2013, 8, 151-158. [CrossRef] [PubMed]

117. Dashti, M.; Scott, J.A.; Edwards, C.A.; Al-Sughayer, M. Predictors of Breastfeeding Duration among Women in Kuwait: Results of a Prospective Cohort Study. Nutrients 2014, 6, 711-728. [CrossRef]

118. Tehranian, A.; Hosseini, L.; Nariman, S.; Eslami, B.; Rastad,H.; Aghajani, F. The Association Between Prepregnancy Body Mass Index and Breastfeeding Duration in Iranian Women. J. Compr. Pediatr. 2017, 8. [CrossRef]

119. Castillo, H.; Santos, I.S.; Matijasevich, A. Maternal pre-pregnancy BMI, gestational weight gain and breastfeeding. Eur. J. Clin. Nutr. 2016, 70, 431-436. [CrossRef]

120. Flores, T.R.; Mielke, G.I.; Wendt, A.; Nunes, B.P.; Bertoldi, A.D. Prepregnancy weight excess and cessation of exclusive breastfeeding: A systematic review and meta-analysis. Eur. J. Clin. Nutr. 2018, 72, 480-488. [CrossRef]

121. Rasmussen, K.M.; Kjolhede, C.L. Prepregnant overweight and obesity diminish the prolactin response to suckling in the first week postpartum. Pediatrics 2004, 113, e465-e471. [CrossRef] [PubMed]

122. Jevitt, C.; Hernandez, I.; Groer, M. Lactation Complicated by Overweight and Obesity: Supporting the Mother and Newborn. J. Midwifery Women's Health 2007, 52, 606-613. [CrossRef] [PubMed]

123. Sejrsen, K.; Purup, S.; Vestergaard, M.; Foldager, J. High body weight gain and reduced bovine mammary growth: Physiological basis and implications for milk yield potential. Domest. Anim. Endocrinol. 2000, 19, 93-104. [CrossRef]

124. Flint, D.J.; Travers, M.T.; Barber, M.C.; Binart, N.; Kelly, P.A. Diet-induced obesity impairs mammary development and lactogenesis in murine mammary gland. Am. J. Physiol. Metab. 2005, 288, E1179-E1187. [CrossRef] [PubMed]

125. Wojcik, K.J.; Mayer-Davis, E.J. Maternal Determinants of Childhood Obesity: Weight Gain, Smoking, and Breastfeeding; Springer: New York, NY, USA, 2010; pp. 93-102.

126. Rasmussen, K.M.; Hilson, J.A.; Kjolhede, C.L. Obesity May Impair Lactogenesis II. J. Nutr. 2001, 131, 3009S-3011S. [CrossRef]

127. Turcksin, R.; Bel, S.; Galjaard, S.; Devlieger, R. Maternal obesity and breastfeeding intention, initiation, intensity and duration: A systematic review. Matern. Child Nutr. 2014, 10, 166-183. [CrossRef]

128. Keely, A.; Lawton, J.; Swanson, V.; Denison, F.C. Barriers to breast-feeding in obese women: A qualitative exploration. Midwifery 2015, 31, 532-539. [CrossRef]

129. LaCoursiere, D.Y.; Baksh, L.; Bloebaum, L.; Varner, M.W. Maternal body mass index and self-reported postpartum depressive symptoms. Matern. Child Health J. 2006, 10, 385-390. [CrossRef]

130. Marseglia, L.; Manti, S.; D’Angelo, G.; Cuppari, C.; Salpietro, V.; Filippelli, M.; Trovato, A.; Gitto, E.; Salpietro, C.; Arrigo, T. Obesity and breastfeeding: The strength of association. Women Birth 2015, 28, 81-86. [CrossRef]

(C) 2020 by the authors. Licensee MDPI, Basel, Switzerland. This article is an open access article distributed under the terms and conditions of the Creative Commons Attribution (CC BY) license (http://creativecommons.org/licenses/by/4.0/). 